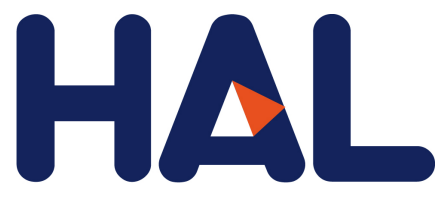

archives-ouvertes

\title{
Building a regime diagram for the Earth's inner core
}

\author{
Marine Lasbleis, Renaud Deguen
}

\section{To cite this version:}

Marine Lasbleis, Renaud Deguen. Building a regime diagram for the Earth's inner core. Physics of the Earth and Planetary Interiors, Elsevier, 2015, 247, pp.80-93. 10.1016/j.pepi.2015.02.001 . hal02304987

\section{HAL Id: hal-02304987 \\ https://hal.archives-ouvertes.fr/hal-02304987}

Submitted on 3 Oct 2019

HAL is a multi-disciplinary open access archive for the deposit and dissemination of scientific research documents, whether they are published or not. The documents may come from teaching and research institutions in France or abroad, or from public or private research centers.
L'archive ouverte pluridisciplinaire HAL, est destinée au dépôt et à la diffusion de documents scientifiques de niveau recherche, publiés ou non, émanant des établissements d'enseignement et de recherche français ou étrangers, des laboratoires publics ou privés. 


\title{
Building a regime diagram for the Earth's inner core
}

\author{
Marine Lasbleis*, Renaud Deguen \\ Laboratoire de Géologie de Lyon LGL-TPE, Université Claude Bernard Lyon 1, École Normale Supérieure de Lyon, CNRS, Lyon, France
}

\section{A R T I C L E I N F O}

Article history:

Received 14 August 2014

Received in revised form 24 January 2015

Accepted 2 February 2015

Available online $\mathrm{xxxx}$

\section{Keywords:}

Inner core

Regime diagram

Seismic anisotropy

Deformation texturing

Viscosity

\begin{abstract}
A B S T R A C T
A number of geodynamical mechanisms have been proposed to explain the origin of the structure of the inner core. Our goal here is to compare quantitatively mechanisms involving deformation of the inner core and build a regime diagram giving the dominant mechanism as a function of key control parameters. A meaningful comparison between the different mechanisms requires the definition of a measure of the strength of the flow, and we choose here to compare this mechanisms in terms of the instantaneous strain rate, and consider that the dominant mechanism is the one which induces the highest strain rates at a given time. Using scaling laws for all proposed mechanisms, we build regime diagrams giving the dominant mechanisms as a function of control parameters including the sign and strength of the density stratification in Earth's inner core, its viscosity, and magnetic field strength. We find that only specific regions of the diagram have a dominant regime potentially consistent with the seismological observed geometry and strength of the inner core anisotropy.
\end{abstract}

\section{Introduction and rationale}

Since the discovery that P-waves in the inner core travel faster along the Earth's spin axis than perpendicular to it (Poupinet et al., 1983), an observation which had soon been interpreted as indicating elastic anisotropy in the inner core (Morelli et al., 1986; Woodhouse et al., 1986), the seismological picture of Earth's inner core has become increasingly complex: the strength and geometry of anisotropy, P-wave velocity, attenuation and anisotropy in attenuation all exhibit radial and longitudinal variations (see Souriau, 2007; Tkalčić and Kennett, 2008; Deuss, 2014; Tkalčić, 2015 for reviews). A number of geodynamical mechanisms have been proposed to explain the origin of the structure of the inner core, with suggested mechanisms falling into two broad categories: (i) solidification texturing, whereby the iron crystals acquire a lattice preferred orientation (LPO) as a result of the solidification process (Karato, 1993; Bergman, 1997), and (ii) development of LPO due to deformation associated with creeping flow in the inner core. Solidification texturing is likely to exist, but to what extent the resulting texture is re-worked by deformation within the inner core is unclear (Bergman, 2002; Bergman et al., 2010; Al-Khatatbeh et al., 2013; Bergman et al., 2014; Lincot et al., 2014). We focus here on deformation mechanisms, with the goal of this study being to compare quantitatively the many mechanisms proposed so far in the literature and build a regime diagram

\footnotetext{
* Corresponding author.
}

giving the dominant mechanism as a function of the values of key control parameters.

It is useful to further subdivide deformation mechanisms between Rayleigh-Bénard-type convection (arising from unstable radial thermal or compositional gradients) and what we will call externally forced mechanisms, with forcing originating from the outer core. Possible forcing include the effect of the core magnetic field, which can drive a flow either through the direct effect of the Lorentz force (Karato, 1999; Buffett and Bloxham, 2000; Buffett and Wenk, 2001) or through heterogeneous Joule heating of the inner core (Takehiro, 2010), and viscous relaxation of a topography of the inner core boundary (ICB) associated with spatially heterogeneous inner core growth (Yoshida et al., 1996).

The existence of Rayleigh-Bénard convection depends on a strict criterion, namely that a Rayleigh number appropriately defined is larger than a critical value. As a consequence, Rayleigh-Bénard convection can exist only under specific conditions. In contrast, externally forced mechanisms can in principle always exist, but the geometry and strength of the flow and associated amount of stress or strain rate then depend on the physical parameters and state of the inner core.

A meaningful comparison between the different proposed mechanisms requires first the definition of a measure of the strength of the flow. Since we are interested in comparing their ability to produce a seismologically observable LPO, possible measures include the instantaneous strain rates or stresses, and cumulative strain. The magnitude of the velocity of the flow is not a good indicator of its ability to produce LPO, which depends on the 
strength of the spatial gradient of the velocity field (deformation rate) - a uniform flow, with therefore no deformation associated, will not produce any texture. The cumulative strain could be a good measure for simple flows, but is difficult to estimate and interpret in the case of time-dependent flows. For this reason, we rather focus on the instantaneous strain rate, and consider that the dominant mechanism at a given time is the one which induces the highest strain rates, when averaged over the inner core. Note that this will not tell us whether the dominant mechanism has the potential to produce an observable seismic anisotropy. For example, large strain rates would not necessarily lead to a significant large scale anisotropy if the flow is highly time-dependent, as is the case of chaotic thermal convection. In addition, the resulting seismic anisotropy depends on the stable phase of iron, its elastic properties and slip system, and the rheology (diffusion creep or dislocation creep), which depends on the level of stress and grain size. Most of this parameters are poorly known at inner core conditions, which makes the process of actually predicting the geometry and strength of the anisotropy well out of the scope of the present paper.

One possible caveat associated with using a global quantity (the averaged strain rate) to discriminate between different mechanisms is that the location of the maximum strain rate may differ among the various deformation mechanisms, which means that it is possible that at a given time different mechanisms are dominant in different parts of the inner core. The complexity of the seismological picture of the inner core may indicate that this indeed the case.

The paper is organized as follow. In Section 2, we discuss under what conditions the inner core has a stable or unstable thermally or compositionally induced density stratification. Scaling laws for the strain rate associated with the various mechanisms proposed in the literature are summarized and discussed in Section 3. The regime diagrams build from these scaling laws are then discussed in Section 4.

\section{Thermal and compositional density stratification}

A first key question for inner core dynamics is whether the radial density profile in the inner core resulting from thermal and compositional variations is stable or unstable. This has been studied in details in a number of previous work (Sumita et al., 1995; Yukutake, 1998; Buffett, 2009; Deguen and Cardin, 2011; Gubbins et al., 2013; Labrosse, 2014). We briefly discuss this question here, following the approach developed in Deguen and Cardin (2011).

A necessary condition for thermal convection to exist is that the temperature profile in the inner core is superadiabatic. It is therefore useful to introduce a potential temperature defined as $\Theta=T(r, \theta, \phi, t)-T_{a d}(r, t)$, where $T_{a d}(r, t)$ is the adiabat anchored at the ICB. The temperature profile is superadiabatic if $\partial \Theta / \partial r>0$, and is subadiabatic if $\partial \Theta / \partial r<0$. With this formulation, the heat equation can be rewritten as

$\frac{D \Theta}{D t}=\kappa_{T} \nabla^{2} \Theta+S_{T}(t), \quad \Theta\left(r_{\text {ic }}\right)=0$,

where $\kappa_{T}$ is the thermal diffusivity (Deguen and Cardin, 2011; Deguen et al., 2013). This formulation of the heat equation remains valid if the temperature variations are not large compared to adiabatic temperature variations, which is the case of the inner core, provided that the dissipation number $D i=\alpha g r_{i c} / c_{p}$ (=0.06 in the inner core) is small (e.g. Tritton, 1988). Though the effective source term $S_{T}(t)$ can include radiogenic heating and Joule heating, it is in fact dominated by the contribution of secular cooling (Yukutake, 1998; Deguen and Cardin, 2011). Ignoring radiogenic and Joule heating, the effective heating rate $S_{T}(t)$ is given by the difference between the rate of heat conduction along the adiabat and secular cooling:

$S_{T}(t)=\kappa_{T} \nabla^{2} T_{a d}-\frac{\partial T_{a d}}{\partial t}$

The term $S_{T}$ is mathematically analog to internal heating. Depending on the sign of $S_{T}$, either an unstable $\left(S_{T}>0\right)$ or stable $\left(S_{T}<0\right)$ thermal stratification can develop in the inner core. The first term on the RHS of Eq. (2) is negative (the inner core loses heat to the outer core) and the term $-\partial T_{a d} / \partial t$ is positive, which shows that the inner core can develop a superadiabatic temperature profile only if the cooling rate at the ICB is fast enough compared to thermal conduction.

The effective heating rate $S_{T}$ can be written as

$S_{T}=3 \frac{\kappa_{T} \rho g^{\prime} \gamma T}{K_{S}}\left[\left(\frac{d T_{s}}{d T_{a d}}-1\right) 2 \frac{r_{\mathrm{ic}}(t)^{2} / 6 \kappa_{T}}{r_{\mathrm{ic}} / u_{\mathrm{ic}}}-1\right]$,

(Deguen and Cardin, 2011), where $r_{\text {ic }}(t)$ is the radius of the inner core at time $t, u_{\mathrm{ic}}=d r_{\mathrm{ic}} / d t$ is the growth rate of the inner core, $\rho$ is the density of the inner core, $g^{\prime}=d g / d r$ is the radial derivative of the acceleration of gravity, $\gamma$ the Gruneisen parameter, $K_{S}$ the isentropic bulk modulus, and $d T_{s} / d T_{\mathrm{ad}}$ is the ratio of the Clapeyron slope to the adiabat. The sign of $S_{T}$ depends on whether the parameter

$\mathcal{T}_{\mathrm{ic}, t}=\frac{1}{2}\left(\frac{d T_{s}}{d T_{a d}}-1\right)^{-1} \frac{r_{\mathrm{ic}} / u_{\mathrm{ic}}}{r_{\mathrm{ic}}^{2} / 6 \kappa_{T}}$,

is larger or smaller than $1 . \mathcal{T}_{\text {ic }, t}$ basically compares the timescale of inner core growth $r_{\mathrm{ic}}(t) / u_{\mathrm{ic}}(t)$ at time $t$ to the timescale of thermal diffusion $r_{\mathrm{ic}}(t)^{2} / 6 \kappa_{T}$ (the factor 6 comes from the spherical geometry of the inner core), with the factor $\left(d T_{s} / d T_{a d}-1\right)$ being a measure of the distance of the initial temperature profile from the adiabat. At first order, the radius of the inner core evolves as $r_{\text {ic }} \propto t^{\alpha}$, with $\alpha=1 / 2$ just after the inner core nucleation (Buffett et al., 1992, 1996). Labrosse (2014) shows that during the history of the inner core, this approximation is still relevant, and that the exponent $\alpha$ is between $1 / 3$ and $1 / 2$. In the special case where the radius of the inner core increases as the square root of time, the quantity $6 \kappa_{T} /\left(r_{\text {ic }} u_{\text {ic }}\right)$ is constant and equal to $2 \tau_{\text {ic }} / \tau_{\kappa}$, where $\tau_{\text {ic }}$ is the age of the inner core and $\tau_{\kappa}=r_{\mathrm{ic}}^{2} /\left(6 \kappa_{T}\right)$ is the timescale of thermal diffusion in the inner core calculated with the current inner core radius. The parameter $\mathcal{T}_{\text {ic }}(t)$ is then constant and equal to

$\mathcal{T}_{\text {ic }}=\left(\frac{d T_{s}}{d T_{\text {ad }}}-1\right)^{-1} \frac{\tau_{\text {ic }}}{\tau_{\kappa}}$.

The parameter $\mathcal{T}_{\text {ic }}$, hereafter called the non-dimensional inner core age, is therefore a good indicator of whether the geotherm in the inner core is subadiabatic or superadiabatic: according to Eq. (3), $S_{T}$ would have been positive (leading to an unstable thermal gradient) for most of inner core history if $\mathcal{T}_{\text {ic }}<1$, and negative for most of inner core history if $\mathcal{T}_{\text {ic }}>1$. With the recent estimates of inner core thermal conductivity above $170 \mathrm{~W} \mathrm{~m}^{-1} \mathrm{~K}^{-1}$ (de Koker et al., 2012; Pozzo et al., 2012; Gomi et al., 2013; Pozzo et al., 2014), giving $\kappa_{T} \gtrsim 1.7 \times 10^{-5} \mathrm{~m}^{-2} \mathrm{~s}^{-1}$, we find that the inner core can be superadiabatic $\left(\mathcal{T}_{\text {ic }}<1\right)$ only if the age of the inner core is smaller than $\simeq 300 \mathrm{My}$ (see Fig. 1 ), which would require a $\mathrm{CMB}$ heat flux larger than 30 TW (Labrosse et al., 2001; Nimmo, 2007; Gomi et al., 2013), which is very unlikely. As a comparison, with the much smaller value of thermal conductivity of $k=36 \mathrm{~W} \mathrm{~m}^{-1} \mathrm{~K}^{-1}$ previously proposed by Stacey and Loper (2007) and Stacey and Davis (2008), the requirement for a superadiabatic temperature profile is that $\tau_{i c}<1.3 \mathrm{~Gy}$, which is much more plausible. 


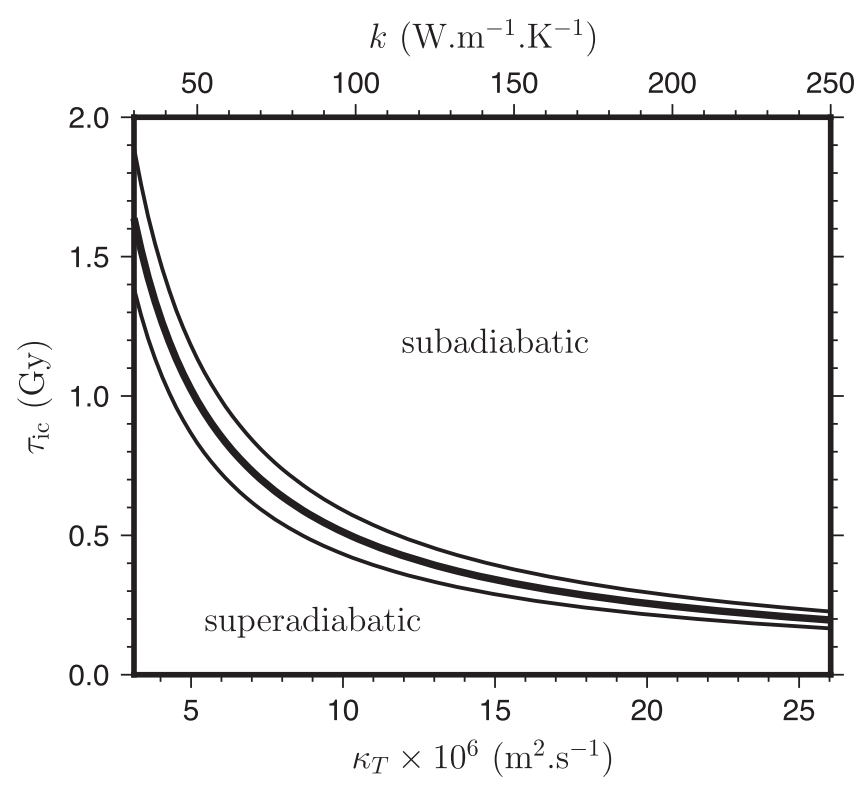

Fig. 1. Critical age of the inner core above which a subadiabatic temperature profile is predicted, as a function of $\kappa$, for $d T_{\mathrm{s}} / d T_{\text {ad }}=1.65 \pm 0.1$. The corresponding value of the thermal conductivity $k$ assuming $\rho=12800 \mathrm{~kg} \mathrm{~m}^{-3}$ and $c_{p}=750 \mathrm{~J} \mathrm{~kg}^{-1}$ is given on the upper axis.

A formulation similar to Eq. (1) can be found for the compositional field (Deguen et al., 2013). We denote by $c^{s}$ and $c^{l}$ the light element concentration in the inner and outer core, respectively, $c_{\text {icb }}^{s, l}$ their values at the ICB, and $\dot{c}_{\mathrm{icb}}^{s, l}=d c_{\mathrm{icb}}^{s, l} / d t$ their time derivatives at the ICB. The concentration in the liquid and solid sides of the ICB are linked by the partition coefficient $D=c_{\mathrm{icb}}^{s} / c_{\mathrm{icb}}^{l}$. Introducing $C=c-c_{\mathrm{icb}}^{s}$, the equation of transport of light elements can be written as

$\frac{D C}{D t}=\kappa_{c} \nabla^{2} C+S_{c}, \quad C\left(r_{\text {ic }}\right)=0$,

where $\kappa_{c}$ is the coefficient of diffusion of the light element and

$S_{c}=-\frac{d c_{\mathrm{icb}}^{s}}{d t}=-D \frac{d c_{\mathrm{icb}}^{l}}{d t}-c_{\mathrm{icb}}^{l} \frac{d D}{d t}$

which is analog to the potential temperature transport Eq. (1). Similarly, the sign of $S_{c}$ determines whether an unstable $\left(S_{c}>0\right)$ or stable $\left(S_{c}<\right)$ compositional stratification would develop in the inner core. In general, the partition coefficient $D$ can vary with time, either because of dynamical effects or because $D$ likely varies with pressure, temperature and composition (Alboussière et al., 2010; Deguen and Cardin, 2011; Gubbins et al., 2013), which can lead to a non-monotonic compositional profile in the inner core (Gubbins et al., 2013; Labrosse, 2014).

One important difference between thermal and compositional stratification comes from the very large difference between thermal and compositional diffusivities. The importance of diffusion can be estimated by comparing the timescales of diffusion of heat or composition, given by $r_{\mathrm{ic}}^{2} /\left(6 \kappa_{T}\right)$ and $r_{\mathrm{ic}}^{2} /\left(6 \kappa_{c}\right)$, to the timescale of inner core growth $r_{\text {ic }} / u_{\text {ic }}$, where $r_{\text {ic }}$ is the radius of the inner core and $u_{\text {ic }}$ its growth rate. The ratio of the timescales of diffusion and inner core growth gives a thermal Péclet number $P e_{T}=u_{\mathrm{ic}} r_{\mathrm{ic}} / \kappa_{T}$ and a compositional Péclet number $P e_{c}=u_{\text {ic }} r_{\text {ic }} / \kappa_{c}$. With a thermal diffusivity $\kappa_{T} \simeq 1.7 \times 10^{-5} \mathrm{~m}^{2} \mathrm{~s}^{-1}$, the diffusion time scale is $\sim 470 \mathrm{My}$. With an age of the inner core $\tau_{\text {ic }}$ between $200 \mathrm{My}$ and $1.5 \mathrm{~Gy}$, the current inner core growth rate is $1.3-9 \times 10^{-11} \mathrm{~m} \mathrm{~s}^{-1}$. The corresponding Péclet values are $P e_{T}=u_{\mathrm{ic}} r_{\mathrm{ic}} / \kappa_{T} \sim 0.9-6$. In contrast, assuming a compositional diffusion coefficient $\kappa_{c} \sim 10^{-12} \mathrm{~m}^{2} \mathrm{~s}^{-1}$ gives a diffusion timescale $\sim 3 \times 10^{7} \mathrm{~Gy}$ and a compositional Péclet number $P e_{c}=u_{\mathrm{ic}} r_{\mathrm{ic}} / \kappa_{c} \sim 10^{7} \gg 1$, which means that the composition is virtually frozen in the inner core.

If the thermal Péclet number is $\mathcal{O}(1)$, then diffusion approximately balances heat production, which gives a potential temperature difference $\simeq S_{T} r_{\text {ic }}^{2} /\left(6 \kappa_{T}\right)$ between the inner core center and the ICB. The density difference associated with potential temperature variations across the inner core is on the order of $\delta \rho_{T}=\alpha_{T} \rho S_{T} r_{\text {ic }}^{2} /\left(6 \kappa_{T}\right) . S_{T}$ is typically on the order of $\pm 100 \mathrm{~K} / \mathrm{Gy}$ (Deguen and Cardin, 2011), which gives a thermally induced density difference across the inner core on the order of $\pm 5 \mathrm{~kg} \mathrm{~m}^{3}$.

If the Péclet number is large and diffusion ineffective, then the difference in concentration is $\int_{0}^{t} S_{c}\left(t^{\prime}\right) d t^{\prime}$ with $t$ the time since inner core nucleation, and the current density stratification is given by $\delta \rho_{c} \sim \alpha_{c} \rho \int_{0}^{\tau_{\text {ic }}} S_{c}\left(t^{\prime}\right) d t^{\prime}$. This can typically be a few $\mathrm{kg} \mathrm{m}^{-3}$, similar to the thermal effect.

In what follows, the strength of the stratification will be measured by a Rayleigh number, which we define as

$R a_{x}=\frac{\delta \rho_{x} g_{\text {icb }} r_{\text {ic }}^{3}}{\eta \kappa_{x}}$

where $\delta \rho_{x}$ is either $\delta \rho_{T}$ in the thermal case or $\delta \rho_{c}$ in the compositional case as defined above.

In this paper, we are interested in the sign and the strength of the stratification, which are both included in the Rayleigh number. A negative Rayleigh number means a stable stratification for which only external forces are relevant and a positive Rayleigh number means either an unstable Rayleigh number if larger than a critical Rayleigh number and the development of convective instabilities, or a stable stratification.

\section{Dynamical regimes}

In this section, we now discuss quantitatively the various published dynamical mechanisms and give scaling laws for the strain rate associated with these mechanisms. We will express the strain rates as functions of a limited number of non-dimensional numbers, summarized in Table 1, to allow easy comparison between the different mechanisms. Several of these non-dimensional numbers have thermal and compositional counterparts (i.e. Rayleigh and Péclet numbers). In the following, we will use a subscript ' $x$ ' denoting either thermal $(x=T)$ or compositional $(x=c)$ parameters in formulas valid for both thermal and compositional fields.

A summary of the different regimes is presented in Fig. 2, with scaling laws for the strain rate and examples of flow produced by these mechanisms. We discuss here the effect of external forcing (heterogeneous growth, Lorentz force and Joule heating) as well as convective instabilities. External forcings exist for both stable and unstable stratification, but specific scaling laws are developed for a strong stable stratification. In this case, we will use the opposite of the Rayleigh number, i.e. the strength of the stable stratification, as the control parameter.

Table 1

A set of non-dimensional numbers for Earth's inner core dynamics.

Non-dimensional number

Rayleigh number

Phase change number

Magnetic number

Péclet number

Inner core growth heterogeneity

$R a_{x}=\frac{\delta \rho_{x} g_{\text {icb }} r_{\text {ic }}^{3}}{\eta \kappa_{x}}$
$\mathcal{P}=\frac{\Delta \rho g_{\text {icb }} r_{\text {ic }} \tau_{\phi}}{\eta}$
$M=\frac{B_{\phi}^{2} r_{\text {ic }}}{\mu_{0} \eta u_{\text {ic }}}$
$P e_{x}=u_{\text {ic }} r_{\text {ic }} / \kappa_{x}$
$S_{2}$

$\mathcal{P}=\frac{\Delta \rho g_{\text {icb }} r_{\text {ic }} \tau_{\phi}}{\eta}$ $P e_{x}=u_{\text {ic }} r_{\text {ic }} / \kappa_{x}$ 


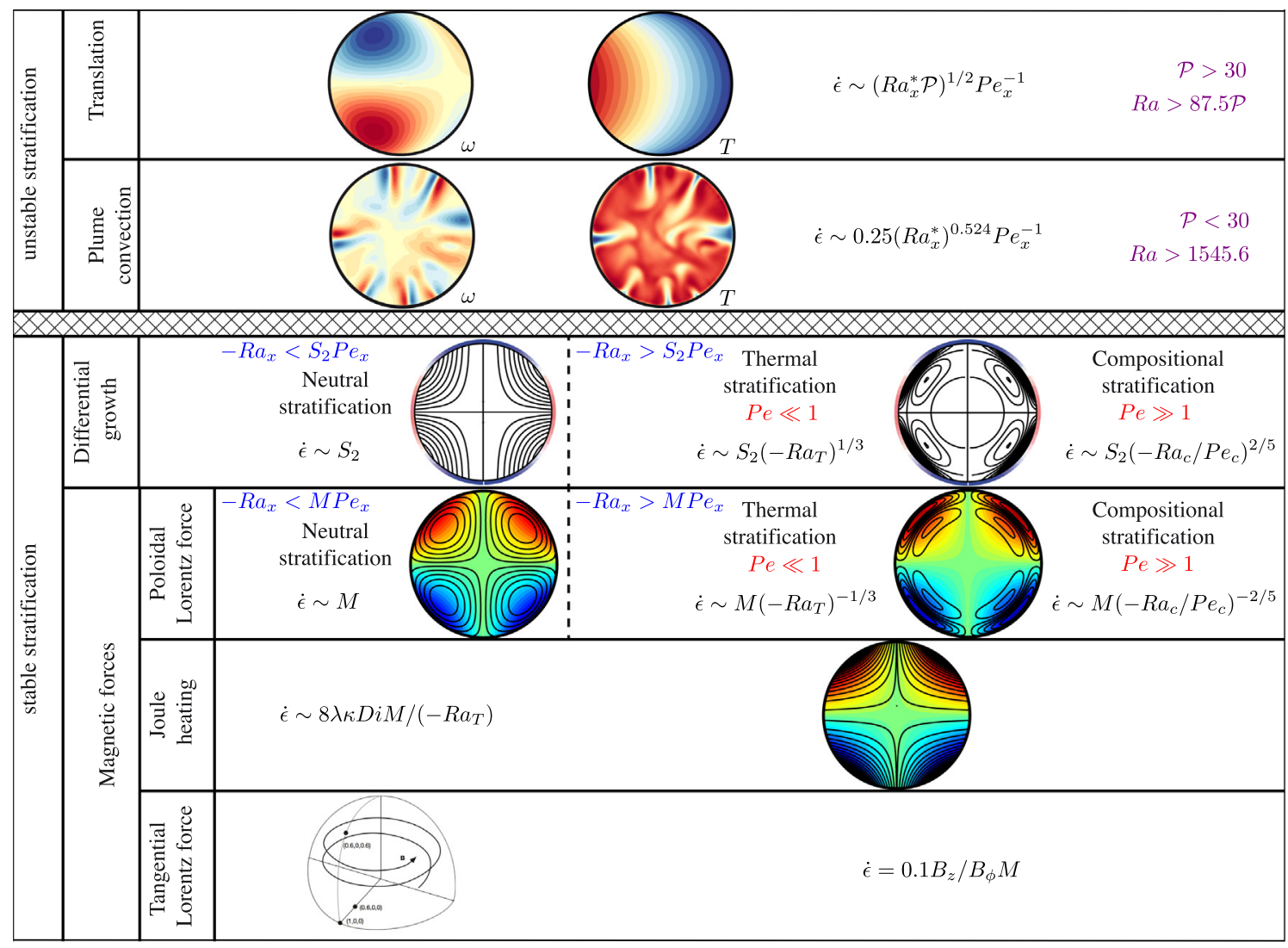

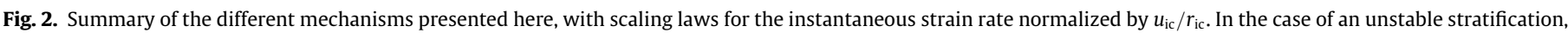

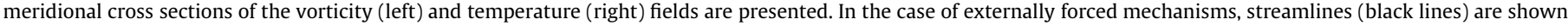
on meridional cross sections, as well as the magnetic field strength in the case of the flow induced by the Lorentz force and heterogeneous Joule heating.

\subsection{Heterogeneous inner core growth and topography relaxation}

Yoshida et al. (1996) have proposed a mechanism based on the idea that the inner core is unlikely to grow in a perfectly spherically symmetric way. The inner core boundary would therefore not exactly coincide with an isopotential surface, and the resulting topographical load would induce horizontal variations in stress within the inner core, which would drive a flow from the high stress regions (below positive topography) to the low stress regions (below negative topography). The process is analog to post-glacial rebound, except that here the topographical load is constantly renewed by solidification. This implies that the system can reach a quasi steady state with a velocity field of magnitude proportional to the amplitude of the solidification rate heterogeneity, provided that the pattern of heterogeneous solidification is maintained on a timescale long compared with the viscous relaxation timescale. For a degree two heterogeneity, the viscous relaxation timescale is $\sim \eta /\left(\Delta \rho g_{\text {icb }} r_{\text {ic }}\right)$, which is at most $\sim 100$ kyrs if $\eta \lesssim 10^{22} \mathrm{~Pa}$ s, and only about one month if the viscosity is, say, $\sim 10^{16}$ Pa s.

Yoshida et al. (1996) argue that the inner core likely grows faster in the equatorial belt than at the poles because of the effect of the Coriolis force on the structure of the flow in the outer core, which is expected to make heat transfer in the outer core anisotropic, with more efficient heat transfer in the directions perpendicular to the Earth's spin axis. Yoshida et al. (1996) therefore assumed a degree 2 , order 0 solidification pattern with a solidification rate of the form $u_{\mathrm{ic}}\left[1-S_{2} P_{2}(\cos \theta)\right]$, where $\theta$ is the colatitude and $P_{2}(x)=\left(3 x^{2}-1\right) / 2$ is the Legendre polynomial of degree 2 . With this formulation, the ratio between the equatorial and polar growth rates is given by $\left(1+S_{2} / 2\right) /\left(1-S_{2}\right)$. Numerical simulations of thermocompositional convection and dynamo action in the core display solidification rates possibly twice larger at the equator than at the poles (e.g. Aubert et al., 2008), which corresponds to $S_{2}=2 / 5$. Additional spatial variations of the solidification rate may be associated with long term control of the mean outer core flow by lateral variations of the heat flux through the core-mantle boundary (Sumita and Olson, 1999; Aubert et al., 2008); this can lead to a deformation pattern in the inner core including degree one and higher degrees components (Deguen, 2012).

In the case of a negligible density stratification, as considered by Yoshida et al. (1996), the flow develops within the whole inner core (see Fig. 2). The velocity simply scales as $S_{2} u_{\text {ic }}$ and varies over a length scale comparable with the inner core radius. The strain rate then scales as

$\dot{\epsilon} \sim \frac{u_{\text {ic }}}{r_{\text {ic }}} S_{2}$

If the inner core is stably stratified, the flow calculated by Yoshida et al. (1996) remains unaltered by the stratification only if $-R a_{T}<S_{2} P e_{T}$ or $-R a_{c}<S_{2} P e_{c}$ (see Appendix A). If the stratification is stronger, then the stable stratification impedes radial motions and the deformation becomes localized in a shear layer below the ICB (see Fig. 2) rather than developing within the whole inner core. To describe this flow, Deguen et al. (2011) used a control parameter defined as

$\mathcal{B}_{x}=\frac{\delta \rho_{x} g_{\text {icb }} r_{\text {ic }}^{2}}{\eta u_{\text {ic }}}$

where $\delta \rho_{x}$ is the difference of density across the ICB associated with either thermal or compositional stratification. In terms of the nondimensional numbers used so far, $\mathcal{B}_{T}=R a_{T} / P e_{T}$ and $\mathcal{B}_{c}=R a_{c} / P e_{c}$. 
The scaling laws for the shear layer thickness $\delta$, for the horizontal velocity $u$ in the layer, and for the strain rate depends on whether the stable stratification is of thermal or compositional origin. In the case of a stable compositional stratification, for which the Péclet number is large and diffusion of the stabilizing field negligible, Deguen et al. (2011) found that the thickness of the shear layer scales as $\delta \sim r_{\text {ic }}\left(-\mathcal{B}_{c}\right)^{-1 / 5}$, while the horizontal velocity in the layer scales as $S_{2} u_{\mathrm{ic}}\left(-\mathcal{B}_{c}\right)^{1 / 5}$. This gives a strain rate

$\dot{\epsilon} \sim \frac{u_{\mathrm{ic}}}{r_{\mathrm{ic}}} S_{2}\left(\frac{-R a_{c}}{P e_{c}}\right)^{2 / 5}$,

where $R a_{c}$ is the Rayleigh number defined in Eq. (8) based on the composition scale $\int_{0}^{t} S_{c} d t^{\prime}$. Notice that the diffusivity $\kappa_{c}$ in $R a_{c}$ and $P e_{c}$ cancel out: the strain rate is independent of the compositional diffusivity.

If thermal stratification is considered instead of compositional stratification, the Péclet number is $\mathcal{O}(1)$ and the scaling given in Eq. (11) does not apply. The correct scaling (demonstrated in Appendix A) is $\delta \sim r_{\text {ic }}\left(-R a_{T}\right)^{-1 / 6}$ and $u \sim S_{2} u_{\text {ic }}\left(-R a_{T}\right)^{1 / 6}$, which gives

$\dot{\epsilon} \sim \frac{u_{\mathrm{ic}}}{r_{\mathrm{ic}}} S_{2}\left(-R a_{T}\right)^{1 / 3}$.

\subsection{Lorentz force}

Poloidal flow. Karato (1999) investigated the effect of the poloidal part of the Lorentz force. Assuming a magnetic field at the inner core boundary of the form $\mathbf{B}=B_{\phi} \cos \theta \sin \theta \mathbf{e}_{\phi}$, with $\theta$ and $\phi$ the colatitude and longitude, the magnitude of the Lorentz force within the inner core is $\sim B_{\phi}^{2} /\left(\mu_{0} r_{\text {ic }}\right)$, where $\mu_{0}=4 \pi 10^{-7} \mathrm{H} \mathrm{m}^{-1}$ is the magnetic permeability of free space and $B_{\phi}$ is the azimuthal component of the magnetic field at the ICB. Assuming a balance between the Lorentz force and the viscous forces, which are $\sim \eta u / r_{\text {ic }}^{2}$, gives a typical flow velocity $\sim B_{\phi}^{2} r_{\text {ic }} /\left(\mu_{0} \eta\right) \sim u_{\text {ic }} M$, where the number $M$ is defined as

$M=\frac{B_{\phi}^{2} r_{\mathrm{ic}}}{\mu_{0} \eta u_{\mathrm{ic}}}$

The typical length scale of the flow is the inner core radius, and the strain rate is

$\dot{\epsilon} \sim \frac{u_{\mathrm{ic}}}{r_{\mathrm{ic}}} M$.

Buffett and Bloxham (2000) argued that if the inner core is stably stratified the poloidal part of the Lorentz force would not produce any significant flow, because a large fraction of the Lorentz force would be balanced by the buoyancy forces arising when isocompositional or isothermal surfaces are deformed. Though the Lorentz force cannot be balanced exactly by the buoyancy forces, the residual flow becomes vanishingly small as the strength of the stratification is increased. Lasbleis et al. (submitted for publication) have shown that the effect of stratification is negligible if $-R a_{T} / P e_{T} \lesssim M$ or $\left|R a_{c}\right| / P e_{c} \lesssim M$. In this case, the scaling law from Eq. 14 is valid.

When the stratification is strong enough to alter the flow, the effect on the pattern of the flow is found to be similar to what was found in the case of heterogeneous inner core growth: radial flow is impeded and the flow is localized in a shear layer below the ICB. Lasbleis et al. (submitted for publication) found that the scaling laws describing the shear layer thickness and the magnitude of the velocity in the layer depend on the value of the Péclet number. In the small Péclet number limit, corresponding to thermal stratification, the thickness $\delta$ of the shear layer scales as $\delta \sim r_{\text {ic }}\left(-R a_{T}\right)^{-1 / 6}$ while the horizontal velocity in the layer scales as $u \sim u_{\mathrm{ic}} M\left(-R a_{T}\right)^{-1 / 2}$. This gives

$\dot{\epsilon} \sim \frac{u_{\text {ic }}}{r_{\text {ic }}} M\left(-R a_{T}\right)^{-1 / 3}$

In the large Péclet number limit, corresponding to compositional stratification, the thickness of the shear layer scales as $\delta \sim r_{\text {ic }}\left(-R a_{c} / P e_{c}\right)^{-1 / 5}$ and $u \sim u_{\text {ic }} M\left(-R a_{c} / P e_{c}\right)^{-3 / 5}$. This gives

$\dot{\epsilon} \sim \frac{u_{\text {ic }}}{r_{\text {ic }}} M\left(\frac{-R a_{\mathrm{c}}}{P e_{\mathrm{c}}}\right)^{-2 / 5}$

As with heterogeneous growth, the scalings are independent of the compositional diffusivity in the case of compositional stratification.

Notice that the shear layer thickness follows the same scaling as does the layer which develops in the case of a flow forced by heterogeneous inner core growth with stable stratification (see Section 3.1). The key difference is that in the case of heterogeneous growth, the flux of mass across the layer is independent of the stratification strength, since it is set by the magnitude of the solidification rate heterogeneities, so that decreasing the shear layer thickness results in larger horizontal velocities. Increasing the strength of the stratification therefore results in higher velocities and smaller length scales, which together imply a larger strain rate magnitude. In the case of the flow induced by the Lorentz force, the horizontal velocity decreases as the strength of the stratification is increased. The velocity decreases with $(-\mathrm{Ra})$ faster than does the layer thickness, which at the end implies than that the strain rate magnitude decreases with increasing stratification.

Azimuthal flow. With the realization that a stable radial stratification would strongly impede the development of a radial flow in the inner core, Buffett and Wenk (2001) turned their attention to the horizontal part of the Lorentz force, which arises from interactions between the azimuthal component and $z$-component of the magnetic field. Adding a constant $B_{z}$ component to the purely toroidal magnetic field defined previously, the $\phi$-component of the Lorentz force drives a longitudinal flow of magnitude

$v_{\phi}=-\frac{1}{10} \frac{B_{z} B_{\phi}}{\mu_{0} \eta} \frac{r^{3}}{r_{\mathrm{ic}}^{2}} \sin \theta$.

Being horizontal, this flow is not affected by the presence of a density stratification (Buffett and Wenk, 2001). The strain rate at $(r, \theta)$ can be calculated as

$\dot{\epsilon}(r, \theta)=\dot{\epsilon}_{r, \phi}(r, \theta)=\frac{1}{10} \frac{B_{z} B_{\phi} r^{2}}{\mu_{0} \eta r_{\mathrm{ic}}^{2}} \sin \theta$.

The maximum value of $\dot{\epsilon}$ is reached at the equator at the ICB, and is

$\dot{\epsilon}_{\max }=\frac{1}{10} \frac{B_{z} B_{\phi}}{\mu_{0} \eta}=\frac{u_{\mathrm{ic}}}{r_{\mathrm{ic}}} \frac{1}{10} \frac{B_{z}}{B_{\phi}} M$

\subsection{Joule heating}

Another possible effect of the magnetic field comes, as proposed by Takehiro (2010), from the Joule heating associated with the electrical currents $\mathbf{j}=\nabla \times \mathbf{B} / \mu_{0}$ diffused in the inner core. The volumetric rate of Joule heating is given by $Q_{j}=\mathbf{j}^{2} / \sigma=$ $|\nabla \times \mathbf{B}|^{2} /\left(\sigma \mu_{0}^{2}\right)$, where $\sigma$ is electrical conductivity. Because $Q_{j}$ depends on the magnetic field intensity, spatial variations in magnetic field strength induces spatial variations of heating rate, and the resulting horizontal variations of temperature induces horizontal variations of density which can drive a flow. Regions with a larger than average rate of Joule heating will tend to rise, while regions with low Joule heating will tend to sink. 
Takehiro (2010) developed a model for the flow induced by Joule heating associated with the degree 2 component of the toroidal field in the case of a stable thermal stratification, and found that the typical velocity is given by

$u \simeq 8 \frac{Q_{j} r_{\text {ic }}}{\rho c_{p} \delta \bar{\Theta}}, \quad$ with $\quad Q_{j}=\frac{B_{\phi}^{2}}{\mu_{0}^{2} \sigma r_{\text {ic }}^{2}}$,

where $\delta \bar{\Theta}$ is the difference of potential temperature between the ICB and the center of the inner core. With $B_{\phi} \sim 10^{-2} \mathrm{~T}$ and $\sigma=1.5 \times 10^{6} \Omega^{-1} \mathrm{~m}^{-1}, Q_{j}$ is about $10^{-11} \mathrm{~W} \mathrm{~m}^{-3}$. The scaling given by Eq. (20) can be found with the following simple calculation. Neglecting thermal diffusion, a parcel of inner core material would see its temperature increase at a rate $Q_{j} /\left(\rho c_{p}\right)$. The density of the parcel will therefore decrease and the parcel will tend to rise with a velocity $u$. However, if a stable thermal stratification is assumed, the parcel will find itself in warmer - and therefore less dense - surroundings. Viewed from the parcel, the rate of change of the potential temperature around the parcel is given by $u \partial \bar{\Theta} / \partial r$. Assuming that the parcel evolves through a succession of equilibrium states at which its temperature remains equal to that of the surrounding (which is a reasonable assumption since this is a creeping flow), equating the rate of temperature increase $Q_{j} /\left(\rho c_{p}\right)$ of the parcel with $u \partial \bar{\Theta} / \partial r \sim u \bar{\Theta} / r_{\text {ic }}$ gives the velocity scaling given in Eq. (20).

The length scale of velocity variations is the radius of the inner core, and the strain rate is therefore

$\dot{\epsilon} \simeq 8 \frac{Q_{j}}{\rho c_{p} \delta \bar{\Theta}} \simeq \frac{u_{\text {ic }}}{r_{\text {ic }}} 8 \frac{\lambda}{\kappa_{T}} \frac{\alpha_{T} g_{\text {icb }} r_{\text {ic }}}{c_{p}} M\left(-R a_{T}\right)^{-1}$,

where $\lambda=1 /\left(\sigma \mu_{0}\right)$. With $\lambda / \kappa \simeq 3 \times 10^{4}$, and the dissipation number $D i=\left(\alpha_{T} g_{\text {icb }} r_{\text {ic }}\right) / c_{p} \simeq 7 \times 10^{-2}$, we have approximately $\dot{\epsilon} \simeq\left(u_{\text {ic }} / r_{\text {ic }}\right) 2 \times 10^{4} M\left(-R a_{T}\right)^{-1}$.

The analysis done by Takehiro (2010) cannot be simply transposed to the case of stable compositional stratification. If locally heated, a parcel of inner core material will rise but, in the case of a stable compositional stratification, will have a concentration in light elements lower than the surroundings. The parcel will stop when the effect of composition on density compensates the effect of temperature. Ignoring thermal diffusion, and starting at a time $t=0$ with no horizontal temperature variations, the temperature excess in a region of high Joule heating at a time $t$ is $\sim Q_{j} t /\left(\rho c_{p}\right)$ and the associated density decrease is $\sim \alpha_{T} \rho Q_{j} t /\left(\rho c_{p}\right) \sim \alpha_{T} Q_{j} t / c_{p}$. Now, if a parcel is displaced upward by an amount $h$, the compositional difference between the parcel and the environment is $\sim(\partial c / \partial r) h$ and the density difference arising from the compositional effect is $\alpha_{c} \rho(\partial c / \partial r) h$. Thermal and compositional effects compensate when

$h \sim \frac{\alpha_{T} Q_{j}}{c_{p} \alpha_{c} \rho(\partial c / \partial r)} t$.

Assuming that the parcel evolves through a succession of equilibrium states at which thermal and compositional effects compensate, the heated part of the inner core would rise at a velocity $u=d h / d t$ given by

$u \sim \frac{\alpha_{T} Q_{j}}{c_{p} \alpha_{c} \rho(\partial c / \partial r)}$.

According to Eqs. (22) and (23) with $Q_{j} \sim 10^{-11} \mathrm{~W} \mathrm{~m}^{-3}$ and $\alpha_{c} \rho(\partial \bar{c} / \partial r) \sim 5 \times 10^{-6} \mathrm{~kg} \mathrm{~m}^{-3}$, the total displacement in $500 \mathrm{My}$ is $\sim 500 \mathrm{~m}$, and the typical velocity is $\sim 10^{-14} \mathrm{~m} \mathrm{~s}^{-1}$, which is negligibly small compared with the other mechanisms. In addition, the assumptions made in this simple calculation were chosen as to maximize the velocity estimate. Including the effect of thermal diffusion in particular would make the process even slower.

\subsection{Rayleigh-Bénard convection}

The last mechanism we consider is Rayleigh-Bénard type convection, i.e. natural convection arising from an unstable radial density gradient. This has been first proposed for the inner core by Jeanloz and Wenk (1988), and later studied in more details (Weber and Machetel, 1992; Wenk et al., 2000; Buffett, 2009; Monnereau et al., 2010; Alboussière et al., 2010; Deguen and Cardin, 2011; Cottaar and Buffett, 2012; Mizzon and Monnereau, 2013; Deguen et al., 2013).

One peculiarity of convection in Earth's inner core is that the inner core boundary is a phase change interface, at which solidification or melting can occur. If the ICB is deformed in response to internal stresses, regions of negative topography will tend to crystallize faster, while regions of positive topography will tend to crystallize at a slower rate or even melt back, depending on the magnitude of the topography. The rate of phase change can be shown to be proportional to the dynamic topography (Alboussière et al., 2010), with a timescale of topography erosion by melting or solidification $\tau_{\phi}$ estimated to $\sim 1000$ years by Alboussière et al. (2010). The behavior of the interface depends on the value of a non-dimensional number, here called the "phase change number", defined as

$\mathcal{P}=\frac{\tau_{\phi}}{\tau_{\eta}}$

where $\tau_{\eta}=\eta /\left(\Delta \rho g_{\text {icb }} r_{\text {ic }}\right)$ is the timescale of viscous relaxation of a topography of wavelength $\sim r_{\text {ic }}$ (see Deguen et al., 2013 for more details on the definition of $\mathcal{P}$ and description of boundary conditions allowing for melting/solidification at the ICB).

In addition to the phase change number $\mathcal{P}$, the style and vigor of convection depends on thermal and compositional Rayleigh numbers. Though thermal and compositional convection, if they exist, are driven respectively by cooling at the ICB and change in composition at the ICB, the two situations are mathematically equivalent to convection driven by an internal buoyancy source, which is either $S_{T}$ or $S_{c}$ as defined in Section 2. Unlike the mechanisms discussed so far, the relevant definition of the Rayleigh number is then

$R a_{x}^{*}=\frac{\alpha_{x} \rho g_{\text {icb }} S_{x} r_{\mathrm{ic}}^{5}}{6 \kappa_{x}^{2} \eta}$

in both the thermal and compositional cases. In the thermal case, the definition is equivalent to the Rayleigh number used so far and $R a_{T}^{*}=R a_{T}$. In the compositional case, the definition is different, and $R a_{c}^{*} \sim R a_{c} / P e_{c}$.

Large $\mathcal{P}$ limit. If the phase change number $\mathcal{P}>30$, dynamically induced melting and solidification happen at a rate small compared with typical convection velocity within the inner core. The inner core boundary then behaves as an impermeable boundary (i.e. there is negligible mass flux across the ICB) and the regime of convection is not affected by phase change at the ICB (Deguen et al., 2013; Mizzon and Monnereau, 2013). The inner core is unstable if the Rayleigh number is larger than a critical value of 1545.6 , above which the first unstable mode has a degree 1 pattern (Chandrasekhar, 1961). Smaller scales modes appear when the Rayleigh number is increased, a chaotic regime with plumes originating from below the ICB being eventually reached (Weber and Machetel, 1992; Deguen and Cardin, 2011; Mizzon and Monnereau, 2013; Deguen et al., 2013).

Deguen et al., 2013 have developed scaling laws for the velocity, mean temperature, horizontal spacing between plumes, and typical strain rates valid for chaotic internally heated convection in a sphere in statistically steady state. In particular, the typical value of the strain rate is found to be 
$\dot{\epsilon} \simeq 0.25 \frac{u_{i c}}{r_{i c}} \frac{R a_{x}^{* 0.524}}{P e_{x}}$

in numerical simulations (Deguen et al., 2013, Table 3). In principle, the scaling laws found by Deguen et al. (2013) are valid for both the thermal and compositional cases, provided that the Rayleigh number is large enough, and that the statistically steady state assumption applies. However, since the inner core is growing and the source terms $S_{T}$ and $S_{c}$ in the heat and composition equations vary with time, whether the inner core can be considered in quasi-steady state is open to question. It has been verified in numerical simulations of thermal convection including inner core growth and core thermal evolution that the scaling laws developed for statistically steady state correctly predict the dynamical state of the inner core (Deguen et al., 2013), but no similar verification has been done in the case of compositional convection.

One way to estimate the approach to quasi-steady state is to compare the timescale needed to reach the mean temperature or composition predicted by the steady-state scaling law, and compare this timescale to the timescale $r_{\text {ic }} / u_{\text {ic }}$ of inner core growth. In steady state, the mean potential temperature and composition are given by $\langle\Theta\rangle=2.9\left(S_{T} r_{\text {ic }}^{2} / 6 \kappa\right) R a_{T}^{*-0.238}$ and $\langle C\rangle=2.9$ $\left(S_{c} r_{\text {ic }}^{2} / 6 \kappa_{c}\right) R a_{c}^{*-0.238}$ (Deguen et al., 2013). The time needed to reach these mean temperature and composition are $\langle\Theta\rangle / S_{T}=2.9$ $\left(r_{\text {ic }}^{2} / 6 \kappa\right) R a_{T}^{*-0.238}$ and $\langle C\rangle / S_{c}=2.9\left(r_{\text {ic }}^{2} / 6 \kappa_{c}\right) R a_{c}^{*-0.238}$, respectively. The ratio of these timescales to the timescale of inner core growth $r_{\text {ic }} / u_{\text {ic }}$ are given by $0.48 P e_{T} R a_{T}^{*-0.238}$ and $0.48 P e_{c} R a_{c}^{*-0.238}$. The Péclet numbers being fixed, the inner core can approach a quasi steady state only if its Rayleigh number is large enough. In the case of thermal convection, $P e_{T}<6$ and a quasi-steady state regime can be reached within Earth's inner core history if $R a_{T}^{*} \gg$ $(0.48 \mathrm{Pe})^{1 / 0.238} \sim 10^{2}$. In the case of compositional convection, $P e_{c} \sim 10^{7}$, and a quasi-steady state regime can be reached if $R a_{c}^{*} \gg\left(0.48 P e_{c}\right)^{1 / 0.238} \sim 10^{28}$. With $S_{c} \sim 10^{-20} \mathrm{~s}^{-1}$, we obtain $R a_{c}^{*} \sim\left(\eta / 10^{16} \mathrm{~Pa} \mathrm{~s}\right) \times 10^{23}$ which, although large, does not allow to reach a quasi-steady state.

Small $\mathcal{P}$ limit. When the phase change number $\mathcal{P}$ is small, phase change at the ICB is fast and profoundly modifies the regime of convection, since a new mode emerges, consisting in a global translation of the inner core with solidification on one hemisphere and melting on the other (Monnereau et al., 2010; Alboussière et al., 2010; Mizzon and Monnereau, 2013; Deguen et al., 2013). At zeroth order in $\mathcal{P}$, the motion is a pure translation with velocity $\kappa / r_{\text {ic }}\left(6 R a_{x}^{*} / 5 \mathcal{P}\right)^{1 / 2}$, with no deformation, and $\dot{\epsilon}=0$. However, since the viscosity of the inner core is finite, deformation is still associated with the translation regime: the horizontal density variations associated with the translation induces a large scale $l=1$ convective flow, with a typical velocity of order $\mathcal{P}$ times the translation velocity (Deguen et al., 2013), which gives a typical velocity $\sim\left(R a_{x}^{*} \mathcal{P}\right)^{1 / 2} \kappa_{x} / r_{\text {ic }}$. The resulting strain rate is

$\dot{\epsilon} \sim \frac{u_{\mathrm{ic}}}{r_{\mathrm{ic}}} \frac{\left(R a_{x}^{*} \mathcal{P}\right)^{1 / 2}}{P e_{x}}$.

This scaling law is valid irrespectively of whether the buoyancy source is of thermal or compositional origin (Deguen et al., 2013).

\section{Regime diagram}

We now compare the instantaneous strain rate of all the different mechanisms discussed in Section 3 to build regime diagrams for inner core dynamics, assuming that the dominant regime for given values of the control parameters is the one with the largest instantaneous strain rate. We normalize the strain rate by $u_{\mathrm{ic}} / r_{\mathrm{ic}}$, and thus a value close to 1 corresponds to a cumulative strain of $\sim 100 \%$ over the whole history of the inner core, had the strain rate been constant and continuously applied on the same parcel.

The inner core viscosity is the parameter with the largest uncertainties, with published estimates ranging from $10^{11}$ to $10^{22} \mathrm{~Pa} \mathrm{~s}$ (Yoshida et al., 1996; Buffett, 1997; Van Orman, 2004; Mound and Buffett, 2006; Koot and Dumberry, 2011; Reaman et al., 2011, 2012; Gleason and Mao, 2013; Davies et al., 2014). $R a_{x}, M$, and $\mathcal{P}$ are inversely proportional to the viscosity, whereas $S_{2}$ and $P$ are independent of $\eta$. The second key point is, as discussed in Section 2, whether the density profile of the inner core is stable or unstable, since it leads to drastically different dynamical regimes.

We will therefore focus on the effect of the viscosity and on the strength and sign of the stratification to construct regime diagrams of the dynamics of the inner core. The relevant measure of the stratification depends on the type of stratification, compositional or thermal, and the two cases will be discussed separately.

Other parameters for the core are known with large uncertainties or have been recently been discussed, such as the intensity of the magnetic field at the vicinity of the inner core boundary and the thermal diffusivity of solid iron at core pressure, and the effect of varying these parameters will be discussed in the text. Typical values for all the parameters are given in Table 2 .

Fig. 2 summarizes the different mechanisms and their requirements in terms of dimensionless parameters. However, we choose here to use a parameter space using dimensional parameters, focusing on the effect of the viscosity and sign and strength of the stratification.

\subsection{Thermal stratification}

As discussed in Section 2, the parameter $\mathcal{T}_{\text {ic }}$ defined in Eq. (5) is a good indicator of the thermal state of the inner core, with $\mathcal{T}_{\text {ic }}>1$ implying a subadiabatic temperature profile and $\mathcal{T}_{\text {ic }}<1$ implying a superadiabatic temperature profile. We will use this parameter as a measure of thermal stratification, and build regime diagrams in $\left(\eta, \mathcal{T}_{\text {ic }}\right)$ space. For a given value of $\mathcal{T}_{\text {ic }}$, the corresponding age of the inner core, $\tau_{\mathrm{ic}}$, can be calculated from Eq. (5) if the inner core radius is assumed to increase as the square root of time. The

Table 2

Parameters used in this study.

\begin{tabular}{lll}
\hline Parameter & Symbol & Value \\
\hline Thermal diffusivity $^{\mathrm{a}}$ & $\kappa_{T}$ & $1.7 \times 10^{-5} \mathrm{~m} \mathrm{~s}^{-2}$ \\
Compositional diffusivity $^{\mathrm{b}}$ & $\kappa_{c}$ & $10^{-12} \mathrm{~m} \mathrm{~s}^{-2}$ \\
Magnetic field strength $^{\mathrm{c}}$ & $B$ & $3 \mathrm{mT}$ \\
Phase change timescale $^{\mathrm{d}}$ & $\tau_{\phi}$ & $10^{3} \mathrm{yrs}^{-3}$ \\
Density jump at the ICB $^{\mathrm{e}}$ & $\Delta \rho$ & $600 \mathrm{~kg} \mathrm{~m}^{-3}$ \\
Radius of ICB $^{\mathrm{e}}$ & $r_{\text {ic }}$ & $1221 \mathrm{~km}^{\mathrm{i}}$ \\
Density of solid iron $^{\mathrm{e}}$ & $\rho$ & $12800 \mathrm{~kg} \mathrm{~m}^{-3}$ \\
Acceleration of gravity $^{\mathrm{e}}$ (at ICB) & $g_{\text {icb }}$ & $4.4 \mathrm{~m} \mathrm{~s}^{-2}$ \\
Thermal expansivity $^{\mathrm{f}}$ & $\alpha$ & $10^{-5} \mathrm{~K}^{-1}$ \\
Heat capacity $^{\mathrm{g}}$ & $C_{p}$ & $750 \mathrm{Jg}^{-1} \mathrm{~K}^{-1}$ \\
Electrical conductivity $^{\mathrm{a}}$ & $\sigma$ & $1.5 \times 10^{6} \mathrm{~S} \mathrm{~m}^{-1}$ \\
Grüneisen parameter $^{\mathrm{f}}$ & $\gamma$ & 1.4 \\
Isentropic bulk modulus $^{\mathrm{e}}$ & $K_{s}$ & $1300 \mathrm{GPa}$ \\
Clapeyron/adiabat slope ratio $^{\mathrm{h}}$ & $\partial T_{\text {ad }} / \partial T_{s}$ & 1.65 \\
\hline
\end{tabular}

a Gomi et al., 2013.

b Gubbins and Davies, 2013.

c Gillet et al., 2010.

d Deguen et al., 2013.

e Dziewoński and Anderson, 1981.

f Vočadlo, 2007.

g Gubbins et al., 2003.

h Deguen and Cardin, 2011. 
conversion depends mostly on the assumed value of the thermal conductivity.

Fig. 3 shows the maximum instantaneous strain rate in the $\left(\eta, \mathcal{T}_{\text {ic }}\right)$ space. A zoom on the boundary between stable and unstable stratification is also provided. For a given set of parameters, the mechanism with the largest instantaneous strain rate is assumed to be dominant, and is shown in Fig. 3. The dashed black lines on Fig. 3 show the boundaries between the different regimes, with the name of the mechanism and a snapshot of the flow shown for each regime.

The stratification is stable whenever the Rayleigh number is lower than the critical Rayleigh number for convection, which is equal to $R a_{\text {limit }}=1545.6$ (Chandrasekhar, 1961) when $\mathcal{P}$ is large (corresponding to low viscosities), and varies with $\mathcal{P}$ as $R a_{\text {limit }}=87.5 \mathcal{P}$ when $\mathcal{P}$ is small (corresponding to large viscosities) (Deguen et al., 2013). This boundary is shown on Fig. 3 in the second panel. With a thermal conductivity equal to $180 \mathrm{~W} \mathrm{~m}^{-1} \mathrm{~K}^{-1}$, the ratio $R a / \mathcal{P}$ reaches 87.5 at $\mathcal{T}_{\text {ic }}=0.9992$, where $\mathcal{T}_{\text {ic }}=1$ corresponds here to $\tau_{\text {ic }}=300$ kyrs. The boundary is independent of $\eta$. In terms of $\mathcal{T}_{\text {ic }}$, the onset of convection always appears very close to the limit $\mathcal{T}_{\text {ic }}=1$, which is why it is not visible on the main panel of Fig. 3.

\subsubsection{Stable stratification}

In the case of stable stratification, all the mechanisms due to external forcing coexist in the inner core. The expected dominant mechanisms for a stable inner core are shown in Fig. 3, for $\mathcal{T}_{\text {ic }}>1$ : deformation induced by heterogeneous growth is predicted to be dominant if the viscosity is larger than $10^{11-12} \mathrm{~Pa}$, while the effect of the azimuthal component of the Lorentz force dominates if the viscosity is lower than this. If the viscosity is

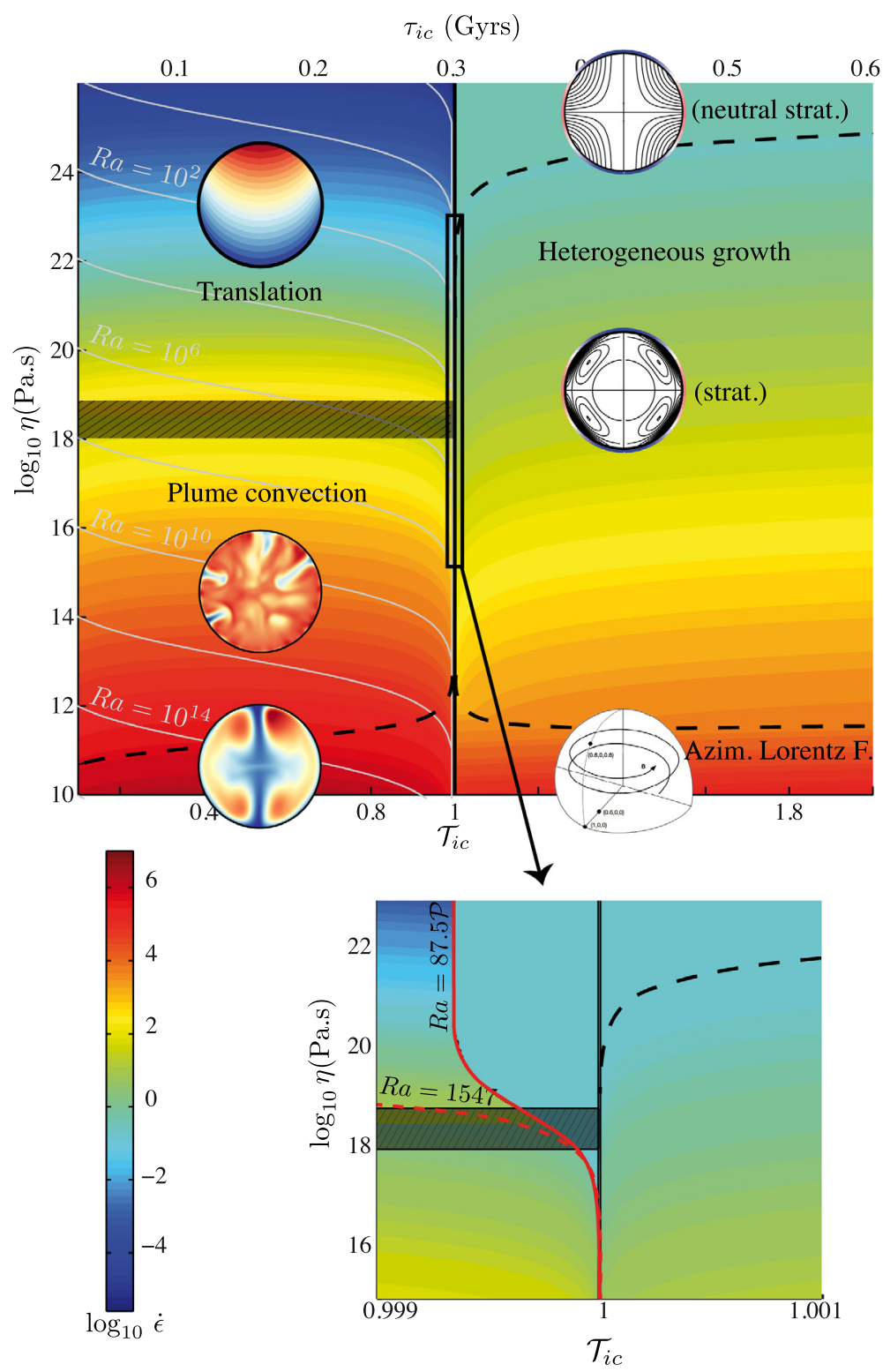

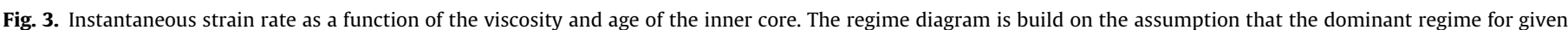

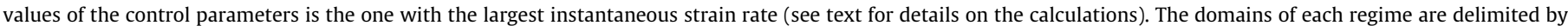

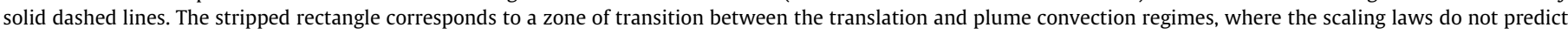

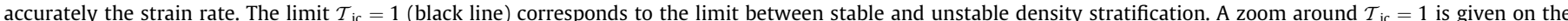

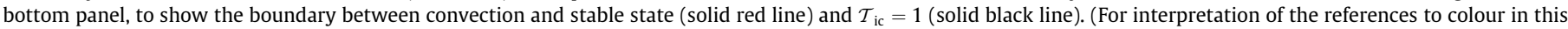
figure legend, the reader is referred to the web version of this article.) 
larger than $10^{23-24} \mathrm{~Pa} \mathrm{~s}, S_{2} P e_{T} /\left|R a_{T}\right| \ll 1$ and a flow similar to the one for neutral stratification (Yoshida et al., 1996) is reached.

Fig. 4 shows the viscosity dependence of the strain rate for all of the mechanisms for $\mathcal{T}_{\text {ic }}=1.65 \quad\left(\kappa_{\text {ic }}=0.5\right.$ Gyrs, $\quad k=180 \mathrm{~W}$ $\mathrm{m}^{-1} \mathrm{~K}^{-1}$ ). The strain rate shown in Fig. 3 is obtained by maximizing the strain rate from Fig. 4. Fig. 4 shows also the effect of a larger magnetic field: with a magnetic field $B=3 \times 10^{-2} \mathrm{~T}$, the boundary between heterogeneous growth rate and azimuthal Lorentz force is shifted toward higher viscosity values $\left(\sim 10^{14} \mathrm{~Pa} \mathrm{~s}\right.$ ) and Joule heating produces a deformation with an intensity similar to heterogeneous growth rate.

\subsubsection{Unstable stratification}

If the Rayleigh number is larger than the critical Rayleigh number, convective instabilities develop. Unlike externally forced mechanisms, these two regimes cannot coexist, and the boundary between the two is plotted on Fig. 3. This boundary was estimated by Deguen et al. (2013) to be at $\mathcal{P}=30$, which corresponds to $\eta=3 \times 10^{18} \mathrm{~Pa}$ s. In the transition zone between the translation and plume convection regimes $(10<\mathcal{P}<100)$, the scaling laws do not predict accurately the strain rate, and we use a striped rectangle to identify this domain.

The strain rate associated with the translation regime at large viscosity is low but non zero because of the secondary flow associated with the lateral temperature variations induced by the translation. The strain rate decreases with increasing viscosity, while the translation velocity is an increasing function of the viscosity.

If the viscosity is low, which corresponds to large value of $\mathcal{P}$, the convective instability develops as plumes and the strain rate is large. However, convection is unsteady and the exact position where the strain is applied varies on time scales several orders of magnitude smaller than the age of the inner core.

External forcings are still active when the stratification is unstable, but they are expected to have a smaller effect than convective instabilities in most of the regime diagram, as shown in Fig. 5. However, if the velocities due to these external forces are large enough compared to convective velocities, we expect the convection pattern to be modified by the forcing. To estimate this, the

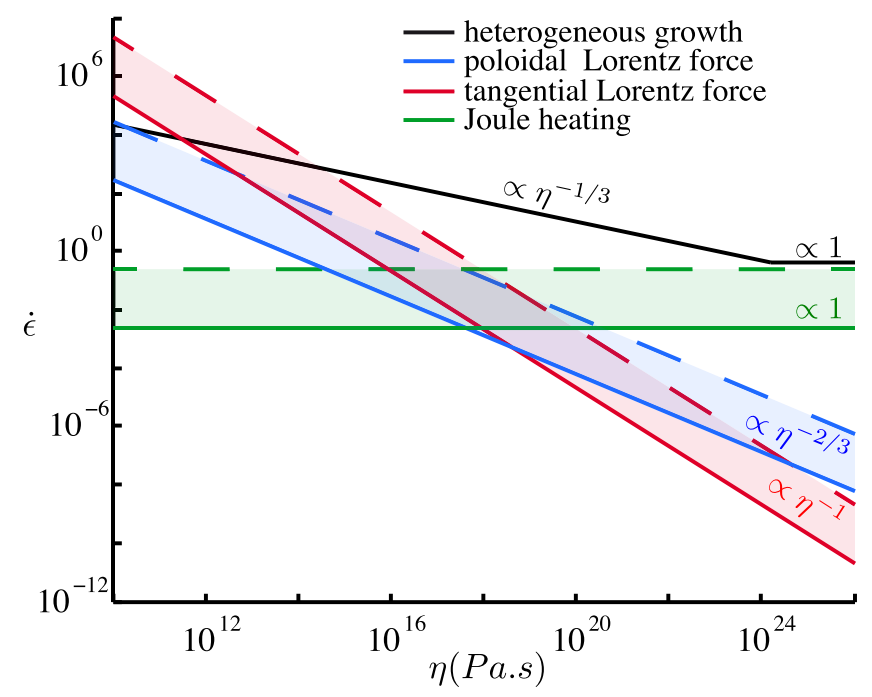

Fig. 4. Stable stratification $\left(\mathcal{T}_{\text {ic }}=1.65\right)$. Instantaneous strain rate as a function of the viscosity. Colors correspond to different mechanisms. For each mechanism, the upper and lower bounds correspond to magnetic field intensities of $B=3 \times 10^{-3} \mathrm{~T}$ and $B=3 \times 10^{-2} \mathrm{~T}$. (For interpretation of the references to colour in this figure legend, the reader is referred to the web version of this article.)

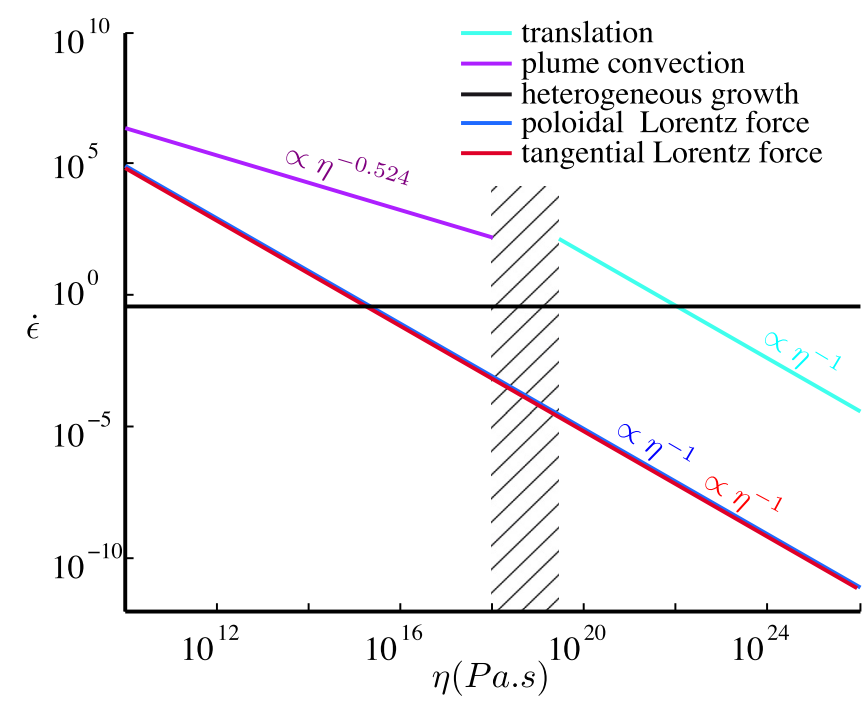

Fig. 5. Unstable stratification $\left(\mathcal{T}_{\text {ic }}=0.5\right)$. Instantaneous strain rate as a function of the viscosity. Colors correspond to different mechanisms. A magnetic field intensity of $B=3 \times 10^{-3} \mathrm{~T}$ is assumed. The stripped rectangle corresponds to a zone of transition between the translation and plume convection regimes $(10<\mathcal{P}<100)$, where the scaling laws do not predict accurately the strain rate. (For interpretation of the references to colour in this figure legend, the reader is referred to the web version of this article.)

typical velocity scales associated with the Lorentz force and preferential growth are compared with the typical velocity of convection. The azimuthal and poloidal magnetic field components promote a flow which velocity scales as $10^{-2} M u_{\text {ic }}$ while the velocity of the flow induced by heterogeneous growth scales as $S_{2} u_{\text {ic }}$ (Yoshida et al., 1996). In the plume convection regime, the velocity scales as $0.96 R a^{* 0.334} / \mathrm{Pe} u_{\text {ic }}$ (Deguen et al., 2013). Magnetic forces will therefore modify the geometry of convection if $M / R a^{* 0.334}$ is larger than $10^{2}$, which requires a low viscosity $\left(\eta<10^{12}\right)$ as seen on the regime diagram of Fig. 3. To obtain a plume velocity of the same order or smaller than $S_{2} u_{\mathrm{ic}}$, the viscosity has to be larger than $10^{21}$, which would put the inner core largely in the translation regime, for which this scaling for the velocity is no longer relevant.

\subsubsection{Effect of thermal diffusivity}

Fig. 6 shows regime diagrams computed with three different values of the thermal diffusivity. The three diagrams are qualitatively similar.

When decreasing the thermal diffusivity and keeping the other parameters unchanged, we obtain slightly larger strain rates and the boundaries between the regimes are shifted toward lower viscosity values, except for the one between translation and plume convection. The effect of the magnetic field is thus larger, for both stably and unstably stratified regions, for a smaller thermal diffusivity.

The main difference between the three diagrams is the dimensional inner core age corresponding to the boundary between the stably and unstably stratified regimes. The limit $\mathcal{T}_{\text {ic }}=1$ corresponds to a dimensional age of the inner core of 1.3 Gyrs if the smallest thermal conductivity $\left(k=36 \mathrm{~W} \mathrm{~K}^{-1} \mathrm{~s}^{-1}\right)$ is assumed, and of less than 0.2 Gyrs for the largest published value $\left(k=232 \mathrm{~W} \mathrm{~K}^{-1} \mathrm{~s}^{-1}\right)$. See Fig. 1 for the evolution of the critical age of the inner core as a function of the conductivity and Section 2 for discussion.

\subsubsection{Exploring the inner core history}

Given the scaling laws for the strain rate induced by the different mechanisms and the time dependence of the dimensionless parameters (assuming the inner core radius evolves as 

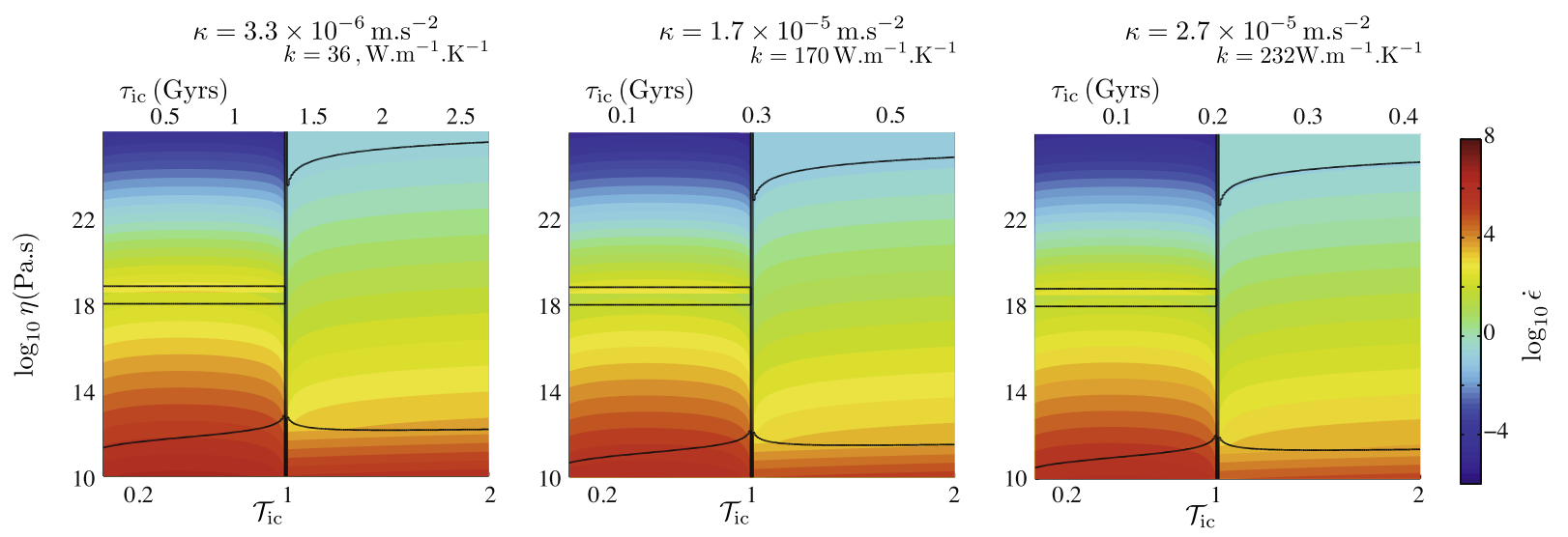

Fig. 6. Regime diagrams for different thermal diffusivities. The strain rate is normalized by $u_{\text {ic }} / r_{\text {ic }}$.

$\left.r_{\text {ic }}(t)=r_{\text {ic }}\left(\tau_{\text {ic }}\right) \sqrt{t / \tau_{\text {ic }}}\right)$ we can investigate how the regime diagram and the strain rate magnitude have involved with time.

We build regime diagrams corresponding to three different times in the history of the inner core, at which the inner core radius is equal to $0.25,0.5$ and 1 times its current radius (Fig. 7). In contrast with Fig. 3, the strain rate is dimensional in this figure, which explains that when decreasing $\mathcal{T}_{\text {ic }}$ in the unstably stratified regime the strain rate is actually increasing, but the total strain over the inner core history would be lower. Using the dimensional strain rate allows us to compare more readily the regime diagrams of this figure.

When going back in time, two main effects are noticeable on the diagrams. First, the stable zone for $\mathcal{T}_{\text {ic }}<1$ is larger. This is because the Rayleigh number is a strong function of $r_{i c}(t)$, and the onset of the instability requires faster inner core cooling (smaller $\mathcal{T}_{\text {ic }}$ ) to compensate for the smaller $r_{i c}$. Even if the set of parameters indicates a translation regime for today's inner core, it is less likely to happen when the inner core was younger. The time at which the translation started depends on the ratio $R a_{T} / \mathcal{P}$ which is proportional to $S\left(\mathcal{T}_{\text {ic }}\right) r_{\text {ic }}^{4}(t)$. Second, the effect of a stable stratification on the flow induced by heterogeneous growth is less pronounced. As discussed in Section 3.1, stratification has a significant effect on the flow induced by heterogeneous growth if $-R a_{T} /\left(S_{2} P e_{T}\right)>1$. Since $-R a_{T} /\left(S_{2} P e_{T}\right) \propto r_{i c}(t)^{6} / \eta$, the limit of the strongly stratified regime is shifted toward smaller values of the viscosity when the inner core radius is smaller, as can be seen in Fig. 7. The effect of stratification is always negligible early in the inner core history, and then becomes increasingly important.
Finally, the $r_{\text {ic }} \propto \sqrt{t}$ growth law assumed here is a special case, for which the source term $S_{T}$ in the heat conservation equation is a constant. In this case, the inner core would remain either stably or unstable stratified during all of its history. If a more realistic growth law (based on core energetics (Labrosse, 2003)) is considered, it can be shown that the source term $S_{T}$ is in general a decreasing function of time, which, even if initially positive, can become negative in the course of inner core solidification (Deguen and Cardin, 2011). This implies that the inner core may have evolved from an unstably stratified regime to a stably stratified regime.

\subsection{Compositional stratification}

Compositional stratification is scaled with the source term $S_{c}=\dot{c}^{S}\left(r_{i c}\right)$, which can be either negative or positive (see Eq. (7)). Values for $-\alpha_{c} S_{c}$ are expected to range from $-10^{-19} \mathrm{~s}^{-1}$ (constant partition coefficient and $\delta \rho=10 \mathrm{~kg} \mathrm{~m}^{-3}$ ) to $+10^{-20} \mathrm{~s}^{-1}$ (from Labrosse (2014) the density variation from oxygen only should be of order $1 \mathrm{~kg} \mathrm{~m}^{-3}$. Considering that sulfur is stabilizing the stratification, this overestimates all reasonable values).

Fig. 8 shows the instantaneous strain rate in a regime diagram in the $\left(\alpha_{c} S_{c}, \eta\right)$ space, build with the same approach as for the thermal case. In the stable stratification case, the mechanism with the maximum instantaneous strain rate is assumed to be dominant and is shown in the figure. Other mechanisms are still valid but are less likely to texture the inner core. In the unstable stratification case, the boundary between the translation and plume
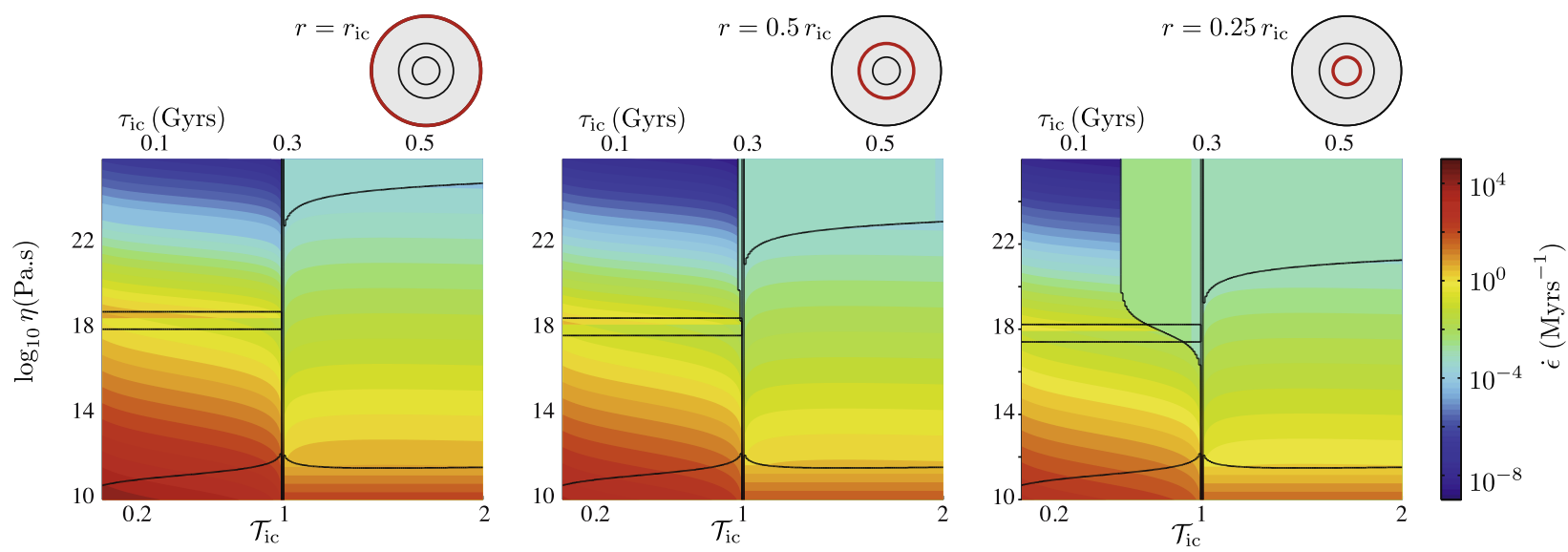

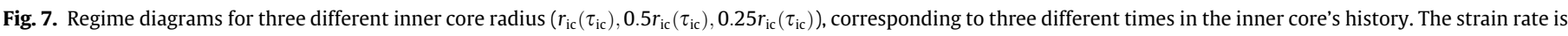
dimensional to enable comparison between the regime diagrams. 


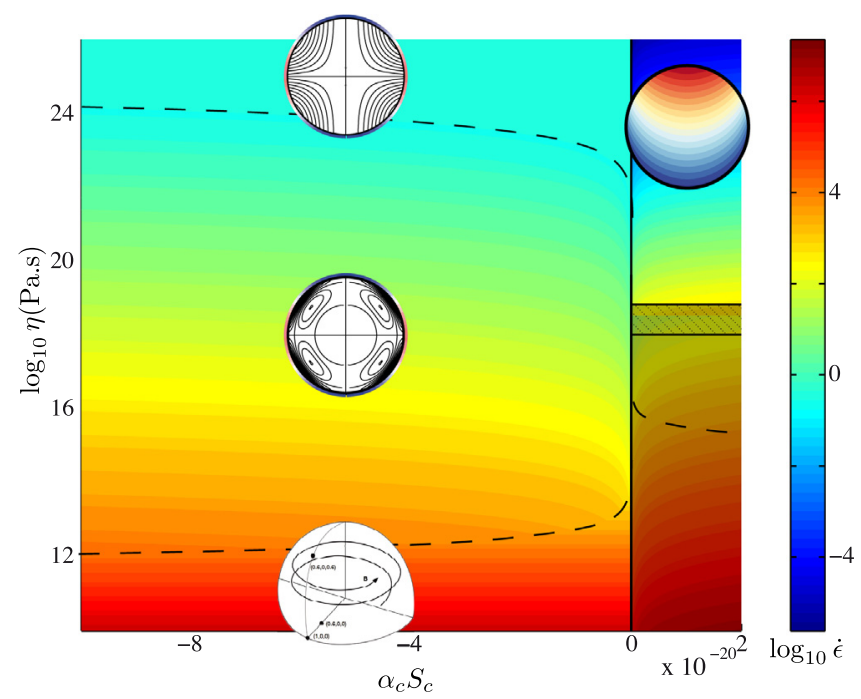

Fig. 8. Regime diagram for compositional stratification. The color intensity shows the instantaneous strain rate and the boundaries between the different regimes are indicated with dashed (coexistence of different regimes) and solid lines (incompatibility between the regimes). For $\alpha_{c} S_{c}>0$ and $\eta<10^{18}$ Pa s (grey shade), convective instabilities develop as plumes but a steady state may not be reached, and the value of the strain rate is uncertain. (For interpretation of the references to colour in this figure legend, the reader is referred to the web version of this article.)

convection regimes is shown, as well as the region where the Lorentz forces are likely to alter the pattern of the flow. As discussed in Section 3.4, it is not clear that the scaling laws developed for plume convection can be used here, as the steady state may not be reached in the case of compositional convection, and we show this domain in the regime diagram with a grey shading. In the plume convection regime, the limit below which the magnetic field can alter the pattern of the flow is also shown.

\section{Discussion and conclusion}

In this paper, we have developed regime diagrams for the dynamics of the inner core based on the instantaneous strain rate associated with each published mechanisms. For a given age and viscosity of the inner core, the flow geometry and the associated strain rate can be inferred from the diagrams. These regime diagrams may also be used to determine which regions in parameter space are likely to correspond to Earth's inner core by comparing the flow geometry and/or the intensity of strain to seismic observations. For the mechanisms discussed here, compositional or thermal stratification have qualitatively similar effects. The scaling laws for the strain rate are different, but for the range of parameters suitable for the Earth's inner core, they both lead to strain rate values of the same order of magnitude.

Developing a significant texture requires that the accumulated strain is at least a few tenths of percents (e.g. Wenk et al., 2000,Deguen et al., 2011). Whether this is likely or not can be roughly estimated from the regime diagrams shown in Figs. 3 and 8 , which show the strain rate normalized by $u_{\text {ic }} / r_{\text {ic }}$, i.e. the dimensional strain rate multiplied by the timescale of inner core growth $r_{\text {ic }} / u_{\text {ic }}$. This gives an estimate of the cumulative strain over the inner core history, had the strain rate remained constant during this time.

Most of the regions of the diagram are expected to produce significant textures when considering the intensity of the strain rate, except for the highest viscosity regions in the translation regime. The translation mode has very large velocities, implying a high replacement rate and a smaller residence time of the crystals in the inner core, which may limit the accumulation of strain. Translation is a good candidate to produce an East-West dichotomy (Monnereau et al., 2010; Alboussière et al., 2010; Bergman et al., 2010; Geballe et al., 2013) but may not produce by itself a texture strong enough to explain the global anisotropy. The regions of the regime diagram with the highest strain rates are found for the lowest viscosity. The instantaneous strain rate in the plume convection regime is large but unlikely to add up because the flow is unsteady. Lorentz force can channel the flow if the viscosity is low enough and may organize the deformation such that the accumulated strain is large enough to be detected by seismic studies.

When considering only the flow geometry, a significant fraction of the mechanisms presented here are unlikely to produce the $\mathrm{N}-\mathrm{S}$ oriented cylindrical symmetry revealed by seismology. Both heterogeneous growth and poloidal Lorentz forcing show such a symmetry if the stratification is weak (Karato, 1999; Yoshida et al., 1996). In the case of a strong stratification (Buffett and Bloxham, 2000; Deguen et al., 2011; Lasbleis et al., submitted for publication), the flow is confined in an upper layer and unlikely to produce the correct anisotropy geometry. The flow induced by the azimuthal Lorentz force (Buffett and Wenk, 2001) may seem unlikely to produce the expected geometry because deformation is restricted to the uppermost inner core, but the accumulated strain in fact increases with depth and reaches a maximum at an intermediate depth (Deguen, 2012). In the case of plume convection, the flow is unsteady and the strain can not add up. If the viscosity is lower than $\sim 10^{12}$ Pa s, magnetic forcing can channel the flow, thus plausibly leading to a cylindrical anisotropy.

A mechanism able to explain the strong anisotropy revealed by seismic data would have to satisfy two requirements: that the induced strain is large enough, and that the flow has the correct geometry. Based on our regime diagram, this seems to require

- either a stable stratification and a viscosity large enough to reach the weakly stratified heterogeneous growth regime, which could give a flow with the adequate geometry (Yoshida et al., 1996). However, the timescale of texture development would be similar to the age of the inner core;

- or a low viscosity ( $\lesssim 10^{12}$ Pa s ) and either a stable or unstable stratification, to allow the Lorentz force to have a strong effect and impose a N-S axisymmetric geometry.

Our regime diagrams still provide only a simplified view of Earth's inner core dynamics. For example, evaluating the effect of thermal and compositional stratification independently is artificial, and ignores the possibility of having double diffusive convection (Deguen and Cardin, 2011; Cottaar and Buffett, 2012; Pozzo et al., 2014). Also, we have limited our analysis to mechanisms with scaling laws for the strain rate available in the literature, and thus our regime diagrams are probably incomplete. In particular, the effect of Joule heating when the inner core is unstable against convection has not yet be investigated, but may be of importance (Takehiro, 2010).

Finally, though the order of magnitude of the cumulative strain is a good indicator of the likelihood of developing a significant texture, predicting travel time residuals requires simulating the texture development process and microscopic properties of iron at inner core pressure and temperature (Lincot et al., 2014).

\section{Acknowledgement}

We thank Stéphane Labrosse for helpful discussions, and two anonymous reviewers for their constructive comments. RD gratefully acknowledges support from grant ANR-12-PDOC-0015-01 of the ANR (Agence Nationale de la Recherche). 


\section{Appendix A. Flow induced by heterogeneous inner core growth in the case of stable thermal stratification}

In this appendix, we derive scaling laws describing the flow forced by heterogeneous growth of the inner core in the case of a stable thermal or compositional stratification. Scaling laws have already been derived by Deguen et al. (2011) in the compositional case, for which the Péclet number is large, but not in the thermal case, for which the Péclet number is on the order of 1 and diffusion is important. In both cases, the stable stratification impedes the development of a strong radial flow because of the buoyancy forces which arise when surface of constant temperature or composition are deformed, and the flow is confined in a shear layer below the ICB. In what follows, we will denote by $\delta$ the thickness of this shear layer, $u_{r}$ and $u_{\theta}$ the radial and latitudinal velocities, and look for scaling laws for these quantities.

We start with the equations of conservation of vorticity assuming axisymmetry around Earth's spin axis (obtained by taking the curl of the Stokes equation), heat, and mass, which we write as

$\mathbf{0}=-\alpha_{x} \rho g^{\prime} \frac{\partial \chi}{\partial \theta}+\eta \nabla^{2} \omega_{\phi}$,

$\frac{D \chi}{D t}=\kappa_{x} \nabla^{2} \chi+S_{x}$

$0=\nabla \cdot \mathbf{u}$

where $\chi$ denotes either the potential temperature $\Theta$ or the modified composition $C=c-c_{\mathrm{icb}}^{s}$ as defined in Section 2, and $x$ denotes either thermal $(x=T)$ or compositional $(x=c)$ parameters, $\mathbf{u}=\left(u_{r}, u_{\theta}\right)$ denotes the radial and latitudinal components of the velocity field, $\omega_{\phi} \mathbf{e}_{\phi}=\nabla \times \mathbf{u}$ the vorticity, with $\mathbf{e}_{\phi}$ the unit vector along the longitudinal direction.

We scale lengths by $r_{\mathrm{ic}}$, time by $\tau_{\mathrm{ic}}$, velocity by $\kappa_{x} / r_{\mathrm{ic}}$, and $\chi$ by $\Delta \chi$.

Assuming for simplicity that $\Delta \chi$ is constant, the system of Eqs. (A.1)-(A.3) is rewritten in non-dimensional form as

$\mathbf{0}=-R a_{x} \frac{\partial \chi}{\partial \theta}+\nabla^{2} \omega_{\phi}$,

$\xi_{x} \frac{\partial \chi}{\partial t}=\nabla^{2} \chi-\left(\mathbf{u}-P e_{x} \mathbf{r}\right) \cdot \nabla \chi+\mathcal{S}_{x}$

$0=\nabla \cdot \mathbf{u}$

where $\mathcal{S}_{x}=S_{x} \tau_{\text {ic }} / \Delta \chi$ and $\xi_{x}=r_{\text {ic }}^{2} /\left(\kappa_{x} \tau_{i c}\right) . \xi_{x}$ is related to $P e_{x}$. For example, $\xi_{x}=2 P e_{x} t$ if the radius of the inner core increases as the square root of time. The term $P e_{\chi} \mathbf{r} \cdot \nabla \chi$ in Eq. (A.5) comes from the time dependence of $r_{\text {ic }}$ (inner core growth), and represents radially inward advection relative to the ICB (e.g. Deguen and Cardin, 2011). With this scaling, the non-dimensional inner core growth rate is given by $u_{\text {ic }} r_{\text {ic }} / \kappa_{\chi}$, which is the Péclet number $P e_{x}$.

We write $\chi=\bar{\chi}+\chi^{\prime}$ with $\bar{\chi}(r, t)$ the potential temperature or composition field for $\mathbf{u}=\mathbf{0}$. $\bar{\chi}$ is solution of

$\xi_{x} \frac{\partial \bar{\chi}}{\partial t}=\nabla^{2} \bar{\chi}+P e_{x} \mathbf{r} \cdot \nabla \bar{\chi}+\mathcal{S}_{x}$

Subtracting Eq. (A.7) to (A.2), and assuming $\chi^{\prime} \ll \bar{\chi}$, we obtain

$\xi_{x} \frac{\partial \chi^{\prime}}{\partial t}=\nabla^{2} \chi^{\prime}-u_{\theta} \frac{\partial \chi^{\prime}}{\partial \theta}-u_{r} \frac{\partial \chi^{\prime}}{\partial r}-u_{r} \frac{\partial \bar{\chi}}{\partial r}+P e_{x} \mathbf{r} \cdot \nabla \chi^{\prime}$.

In what follows, we will look for steady state scaling laws and we will therefore assume that $\xi_{x} \partial \chi^{\prime} / \partial t=0$.

The continuity equation implies $u_{\theta} \sim u_{r} / \delta$. The radial velocity is imposed by the boundary condition, and is on the order of $u_{r} \sim S_{2} u_{\text {ic }} \sim S_{2} P e_{x}$, which gives

$u_{\theta} \sim \frac{S_{2} P e_{x}}{\delta}$.
In the vorticity equation, the two terms must balance when the effect of stratification is important: starting with no perturbations, $\chi^{\prime}=0$, surfaces of constant $\chi$ are deformed by the flow induced by the boundary conditions. This induces an increase of $\chi^{\prime}$ and of the buoyancy force which eventually balances the flow if the stratification is strong enough. With the vorticity $\omega_{\phi} \sim u_{\theta} / \delta \sim S_{2} P e_{x} / \delta^{2}$, we therefore have

$\frac{S_{2} P e_{x}}{\delta^{4}} \sim\left|R a_{x}\right| \chi^{\prime}$.

The scaling of the different terms of the potential temperature/composition equation depends on whether a thermal or compositional stratification is assumed, and will consider both cases separately, starting with the thermal stratification case.

Boundary between weak and strong stratification regimes. The effect of the stratification is negligible if the buoyancy term $\left|R a_{x}\right| \partial \chi^{\prime} / \partial \theta$ cannot balance the viscous forcing term. With $\chi^{\prime}$ being necessarily smaller than the temperature difference across the inner core, which is $\mathcal{O}(1)$ in non-dimensional units in the thermal case, the buoyancy term magnitude is $\lesssim\left|R a_{x}\right|$. The viscous term $\nabla^{2} \omega_{\phi} \sim \omega_{\phi} / \delta^{2} \sim u_{\theta} / \delta^{3} \sim S_{2} P e_{x} / \delta^{4}$ being larger than $S_{2} P e_{x}$, the effect of stratification would therefore be negligible if $S_{2} P e_{x}>\left|R a_{x}\right|$.

\section{A.1. Small Pe limit - thermal stratification}

Scaling of the heat equation terms. With the radial gradient of $\Theta^{\prime}$ scaling as $\Theta^{\prime} / \delta$, the horizontal gradient of $\Theta^{\prime}$ scaling as $\Theta^{\prime}$, and $\partial \bar{\Theta} / \partial r \sim 1$, the different terms of the potential temperature perturbation equation then scale as follows:

$0=\underbrace{\nabla^{2} \Theta^{\prime}}_{\sim \Theta^{\prime} / \delta^{2}}-\underbrace{u_{\theta} \frac{\partial \Theta^{\prime}}{\partial \theta}}_{\sim u_{\theta} \Theta^{\prime}}-\underbrace{u_{r} \frac{\partial \Theta^{\prime}}{\partial r}}_{\sim u_{r} \Theta^{\prime} / \delta}-\underbrace{u_{r} \frac{\partial \bar{\Theta}}{\partial r}}_{\sim u_{r}}+\underbrace{P e_{T} \mathbf{r} \cdot \nabla \Theta^{\prime}}_{\sim P e_{T} \Theta^{\prime} / \delta}$

With $\Theta^{\prime} \sim S_{2} P e_{T} /\left(R a \delta^{4}\right)$ from Eq. (A.10), $u_{r} \sim S_{2} P e_{T} \quad$ and $u_{\theta} \sim S_{2} P e_{T} / \delta$, we obtain

$0=\underbrace{\left.\nabla^{2} \Theta^{\prime}\right)}_{\sim S_{2} P e_{T} /(R a}-\underbrace{u_{\theta} \frac{\partial \Theta^{\prime}}{\partial \theta}}_{\sim S_{2} P e_{T} \Theta^{\prime} / \delta}-\underbrace{u_{r} \frac{\partial \Theta^{\prime}}{\partial r}}_{\sim S_{2} P e_{T} \Theta^{\prime} / \delta}-\underbrace{u_{r} \frac{\partial \bar{\Theta}}{\partial r}}_{\sim S_{2} P e_{T}}+\underbrace{P e_{T} \mathbf{r} \cdot \nabla \Theta^{\prime}}_{\sim S_{2} P e_{T}^{2} /\left(R a \delta^{5}\right)}$.

The advection term must be balanced by either the diffusion term $\nabla^{2} \Theta^{\prime}$, or by the inner core growth term $P e_{T} \mathbf{r} \cdot \nabla \Theta^{\prime}$ (the last term is always smaller than $P e_{T} \mathbf{r} \cdot \nabla \Theta^{\prime}$ since $\left.\delta<1\right)$. Comparing the diffusion term, which is $\sim S_{2} P e_{T} /\left(|R a| \delta^{6}\right)$, with the inner core growth term, which is $S_{2} P e_{T}^{2} /\left(|R a| \delta^{5}\right)$, we find that the effect of inner core growth is negligible if $P e_{T} \ll 1 / \delta$.

Neglecting the term in $P e_{T}$, the equation for the perturbation of the potential temperature writes

$0=\underbrace{\nabla^{2} e_{T} /\left(R a_{T} \delta^{6}\right)}_{\sim S_{2}}-\underbrace{u_{\theta} \frac{\partial \Theta^{\prime}}{\partial \theta}}_{\sim S_{2} P_{P e_{T}} \Theta^{\prime} / \delta}-\underbrace{u_{r} \frac{\partial \Theta^{\prime}}{\partial r}}_{\sim S_{2} P e_{T} \Theta^{\prime} / \delta}-\underbrace{u_{r} \frac{\partial \bar{\Theta}}{\partial r}}_{\sim S_{2} P e_{T}}$.

We now assume that the terms of advection of the temperature perturbation are small compared to the term of vertical advection of the mean temperature profile, which requires that $\Theta^{\prime} / \delta=S_{2} P e_{T} /\left(\left|R a_{T}\right| \delta^{5}\right) \ll 1$, or that $\delta \gg\left(S_{2} P e_{T} /\left|R a_{T}\right|\right)^{1 / 5} \quad$ (selfconsistency will be checked later). Balancing vertical advection and diffusion, we obtain

$\delta \sim\left|R a_{T}\right|^{-1 / 6}$

With $u_{\theta} \sim S_{2} P e_{T} / \delta$, this yields

$u_{\theta} \sim S_{2} P e_{T}\left|R a_{T}\right|^{1 / 6}$ 
and

$\dot{\epsilon} \sim \frac{u_{\theta}}{\delta} \sim S_{2} P e_{T}\left|R a_{T}\right|^{1 / 3}$

The dimensional strain rate is given by

$\dot{\epsilon} \sim \frac{\kappa_{T}}{r_{\text {ic }}^{2}} S_{2} P e_{T}\left|R a_{T}\right|^{1 / 3} \sim \frac{u_{\text {ic }}}{r_{\text {ic }}} S_{2}\left|R a_{T}\right|^{1 / 3}$.

The requirement that $\delta \gg\left(S_{2} P e_{T} /\left|R a_{T}\right|\right)^{1 / 5}$ for horizontal advection to be small compared with vertical advection requires $\left|R a_{T}\right| \gg\left(S_{2} P e_{T}\right)^{6 / 11}$, which is verified here since $P e_{T}$ and $S_{2}$ are both on the order of 1 , while $\left|R a_{T}\right|$ is typically $\gg 1$.

Using the scaling found for $\delta$, the requirement that $P e_{T} \ll 1 / \delta$ for inner core growth to be of negligible effect is written as

$P e_{T} \ll\left|R a_{T}\right|^{1 / 6}$.

\section{A.2. Large Pe limit - compositional stratification}

Scaling of the terms of the composition transport equation. The diffusion term is now assumed to be negligible, which gives

$0=-\underbrace{u_{\theta} \frac{\partial C^{\prime}}{\partial \theta}}_{\sim S_{2} P e_{c} C^{\prime} / \delta}-\underbrace{u_{r} \frac{\partial C^{\prime}}{\partial r}}_{\sim S_{2} P e_{c} C^{\prime} / \delta}-\underbrace{u_{r} \frac{\partial \bar{C}}{\partial r}}_{\sim S_{2} P e_{c}}+\underbrace{P e_{c} \mathbf{r} \cdot \nabla C^{\prime}}_{\sim P e_{c} C^{\prime} / \delta}$.

Assuming again that the composition perturbation advection terms are small compared to the vertical advection of the mean compositional profile, the main balance is between the second and third terms, which gives

$S_{2} P e_{c} \sim \frac{P e_{c} C^{\prime}}{\delta}$

Using $C^{\prime} \sim S_{2} P e_{c} /\left(\delta^{4} R a_{c}\right)$ from Eq. (A.10), we obtain

$\delta \sim\left(\left|R a_{c}\right| / P e_{c}\right)^{-1 / 5}$.

With $u_{\theta} \sim S_{2} P e_{c} / \delta$, this yields

$u_{\theta} \sim S_{2} P e_{c}\left(\left|R a_{c}\right| / P e_{c}\right)^{1 / 5} \sim S_{2} P e_{c}^{4 / 5}\left|R a_{c}\right|^{1 / 5}$,

and

$\dot{\epsilon} \sim \frac{u_{\theta}}{\delta} \sim S_{2} P e_{c}\left(\left|R a_{c}\right| / P e_{c}\right)^{2 / 5} \sim S_{2} P e_{c}^{3 / 5}\left|R a_{c}\right|^{2 / 5}$.

The dimensional strain rate is given by

$\dot{\epsilon} \sim \frac{u_{\text {ic }}}{r_{\text {ic }}} S_{2}\left(\left|R a_{c}\right| / P e_{c}\right)^{2 / 5}$,

as found by Deguen et al., 2011.

Using the scalings found for $\delta$ and $\boldsymbol{u}_{\theta}$, the requirement that $\delta \gg\left(S_{2} P e_{c} /\left|R a_{c}\right|\right)^{1 / 5}$ for the vertical advection term to dominate over the horizontal advection term requires that $S_{2} \ll 1$.

\section{References}

Al-Khatatbeh, Y., Bergman, M.I., Lewis, D.J., Mason, Z., Zhu, L., Rosenstock, S., 2013. Annealing of directionally solidified alloys revisited: no loss of solidification texture in Earth's inner core. Phys. Earth Planet. Inter. 223, 32-39.

Alboussière, T., Deguen, R., Melzani, M., 2010. Melting-induced stratification above the Earth's inner core due to convective translation. Nature 466 (7307), 744-U9.

Aubert, J., Amit, H., Hulot, G., Olson, P., 2008. Thermochemical flows couple the Earth's inner core growth to mantle heterogeneity. Nature 454 (7205), 758-761.

Bergman, M.I., 1997. Measurements of electric anisotropy due to solidification texturing and the implications for the Earth's inner core. Nature 389 (6646), 60-63.

Bergman, M.I., 2002. Preferred crystal orientations due to melt convection during directional solidification. J. Geophys. Res. 107 (B9), 2201.

Bergman, M.I., Al-Khatatbeh, Y., Lewis, D.J., Shannon, M.C., 2014. Deformation of directionally solidified alloys: evidence for microstructural hardening of earth's inner core? C.R. Geosci. 346 (5-6), 140-147.
Bergman, M.I., Lewis, D.J., Myint, I.H., Slivka, L., Karato, S.-I., Abreu, A., 2010. Grain growth and loss of texture during annealing of alloys, and the translation of Earth's inner core. Geophys. Res. Lett. 37 (22), L22313.

Buffett, B., Huppert, H., Lister, J., Woods, A., 1996. On the thermal evolution of the earths core. J. Geophys. Res. 101, 7989-8006.

Buffett, B.A., 1997. Geodynamic estimates of the viscosity of the Earth's inner core. Nature 388 (6642), 571-573.

Buffett, B.A., 2009. Onset and orientation of convection in the inner core. Geophys. J. Int. 179 (2), 711-719.

Buffett, B.A., Bloxham, J., 2000. Deformation of Earth's inner core by electromagnetic forces. Geophys. Res. Lett. 27 (24), 4001-4004.

Buffett, B.A., Huppert, H.E., Lister, J.R., Woods, A.W., 1992. Analytical model for solidification of the Earth's core. Nature 356 (6367), 329-331.

Buffett, B.A., Wenk, H.R., 2001. Texturing of the Earth's inner core by Maxwell stresses. Nature 413 (6851), 60-63.

Chandrasekhar, S., 1961. Hydrodynamic and Hydromagnetic Stability. Oxford University Press.

Cottaar, S., Buffett, B., 2012. Convection in the Earth's inner core. Phys. Earth Planet. Inter. 198-199 (C), 67-78.

Davies, C.J., Stegman, D.R., Dumberry, M., 2014. The strength of gravitational coremantle coupling. Geophys. Res. Lett. 41 (11), 3786-3792.

de Koker, N., Steinle-Neumann, G., Vlček, V., 2012. Electrical resistivity and thermal conductivity of liquid Fe alloys at high P and T, and heat flux in Earth's core. Proc. Nat. Acad. Sci. 109 (11), 4070-4073.

Deguen, R., 2012. Structure and dynamics of Earth's inner core. Earth Planet. Sci. Lett. 333-334 (C), 211-225.

Deguen, R., Alboussière, T., Cardin, P., 2013. Thermal convection in Earth's inner core with phase change at its boundary. Geophys. J. Int. 194 (3), $1310-1334$.

Deguen, R., Cardin, P., 2011. Thermochemical convection in Earth's inner core. Geophys. J. Int. 187 (3), 1101-1118.

Deguen, R., Cardin, P., Merkel, S., Lebensohn, R.A., 2011. Texturing in Earth's inner core due to preferential growth in its equatorial belt. Phys. Earth Planet. Inter. 188 (3-4), 173-184.

Deuss, A., 2014. Heterogeneity and anisotropy of Earth's inner core. Annu. Rev. Earth Planet. Sci. 42 (1), 103-126.

Dziewoński, A.M., Anderson, D.L., 1981. Preliminary reference Earth model. Phys. Earth Planet. Inter. 25 (4), 297-356.

Geballe, Z.M., Lasbleis, M., Cormier, V.F., Day, E.A., 2013. Sharp hemisphere boundaries in a translating inner core. Geophys. Res. Lett. 40 (9), 17191723.

Gillet, N., Jault, D., Canet, E., Fournier, A., 2010. Fast torsional waves and strong magnetic field within the Earth's core. Nature 465 (7294), 74-77.

Gleason, A.E., Mao, W.L., 2013. Strength of iron at core pressures and evidence for a weak earth's inner core. Nat. Geosci. 6, 571-574.

Gomi, H., Ohta, K., Hirose, K., Labrosse, S., Caracas, R., Verstraete, M.J., Hernlund, J.W., Nov., 2013. The high conductivity of iron and thermal evolution of the Earth's core. Phys. Earth Planet. Inter. 224 (C), 88-103.

Gubbins, D., Alfè, D., Davies, C.J., 2013. Compositional instability of earth's solid inner core. Geophys. Res. Lett. 40 (6), 1084-1088.

Gubbins, D., Alfè, D., Masters, G., Price, G., Gillan, M., 2003. Can the Earth’s dynamo run on heat alone? Geophys. J. Int. 155 (2), 609-622.

Gubbins, D., Davies, C.J., 2013. The stratified layer at the core-mantle boundary caused by barodiffusion of oxygen, sulphur and silicon. Phys. Earth Planet. Inter. 215, 21-28.

Jeanloz, R., Wenk, H.R., 1988. Convection and anisotropy of the inner core. Geophys. Res. Lett. 15 (1), 72-75.

Karato, S., 1993. Inner core anisotropy due to the magnetic field-induced preferred orientation of iron. Science 262 (5140), 1708-1711.

Karato, S., 1999. Seismic anisotropy of the Earth's inner core resulting from flow induced by Maxwell stresses. Nature 402 (6764), 871-873.

Koot, L., Dumberry, M., 2011. Viscosity of the Earth's inner core: constraints from nutation observations. Earth Planet. Sci. Lett. 308 (3-4), 343-349.

Labrosse, S., 2003. Thermal and magnetic evolution of the Earth's core. Phys. Earth Planet. Inter. 140 (1), 127-143.

Labrosse, S., 2014. Thermal and compositional stratification of the inner core. C.R. Geosci. 346 (5-6), 119-129.

Labrosse, S., Poirier, J., Le Mouel, J., 2001. The age of the inner core. Earth Planet. Sci. Lett. 190 (3-4), 111-123.

Lasbleis, M., Deguen, R., Cardin, P., Labrosse, S., submitted for publication. Inner core dynamics induced by the Lorentz force. Geophysical Journal International.

Lincot, A., Deguen, R., Merkel, S., Cardin, P., 2014. Seismic response and anisotropy of a model hcp iron inner core. C.R. Geosci. 346 (5-6), 148-157.

Mizzon, H., Monnereau, M., 2013. Implication of the lopsided growth for the viscosity of Earth's inner core. Earth Planet. Sci. Lett. 361, 391-401.

Monnereau, M., Calvet, M., Margerin, L., Souriau, A., 2010. Lopsided growth of Earth's inner core. Science 328 (5981), 1014-1017.

Morelli, A., Dziewoński, A.M., Woodhouse, J.H., 1986. Anisotropy of the inner core inferred from PKIKP travel times. Geophys. Res. Lett. 13 (13), 1545-1548.

Mound, J.E., Buffett, B.A., 2006. Detection of a gravitational oscillation in length-ofday. Earth Planet. Sci. Lett. 243 (3-4), 383-389.

Nimmo, F., 2007. Energetics of the Core. Treatise Geophys. 8, 31-66.

Poupinet, G., Pillet, R., Souriau, A., 1983. Possible heterogeneity of the Earth's core deduced from PKIKP travel times. Nature 305, 204-206.

Pozzo, M., Davies, C., Gubbins, D., Alfè, D., 2012. Thermal and electrical conductivity of iron at Earth/'s core conditions. Nature 485 (7398), 355-358. 
Pozzo, M., Davies, C., Gubbins, D., Alfè, D., 2014. Thermal and electrical conductivity of solid iron and iron-silicon mixtures at Earth's core conditions. Earth Planet. Sci. Lett. 393, 159-164.

Reaman, D., Colijn, H., Yang, F., Hauser, A., Panero, W., 2012. Interdiffusion of earth's core materials to $65 \mathrm{GPa}$ and $2200 \mathrm{k}$. Earth Planet. Sci. Lett. 349, 8-14.

Reaman, D.M., Daehn, G.S., Panero, W.R., 2011. Predictive mechanism for anisotropy development in the Earth's inner core. Earth Planet. Sci. Lett. 312 (3-4), 437442.

Souriau, A., 2007. The earth's cores. Treatise Geophys. 1.

Stacey, F., Loper, D., 2007. A revised estimate of the conductivity of iron alloy at high pressure and implications for the core energy balance. Phys. Earth Planet. Inter. $161,13-18$.

Stacey, F.D., Davis, P.M., 2008. Physics of the Earth. Cambridge University Press.

Sumita, I., Olson, P., 1999. A laboratory model for convection in Earth's core driven by a thermally heterogeneous mantle. Science 286, 1547-1549.

Sumita, I., Yoshida, S., Hamano, Y., Kumazawa, M., 1995. A model for the structural evolution of the earth's core and its relation to the observations. In: Yukutake, T. (Ed.), The Earth's Central Part : Its Structure and Dynamics. Terra Scientific Publishing Company, 232, p. 260.

Takehiro, S.-I., 2010. Fluid motions induced by horizontally heterogeneous Joule heating in the Earth's inner core. Phys. Earth Planet. Inter. 184 (3-4), 134-142.
Tkalčić, H., 2015. Complex inner core of the earth: the last frontier of global seismology. Rev. Geophys., URL <http://dxdoi.org/10.1002/2014RG000469>.

Tkalčić, H., Kennett, B.L.N., 2008. Core structure and heterogeneity: a seismological perspective. Aust. J. Earth Sci. 55 (4), 419-431, URL <http://dx.doi.org/10.1080/ 08120090801888578>.

Tritton, D., 1988. Physical Fluid Dynamics. Clarendon Press, Oxford.

Van Orman, J.A., 2004. On the viscosity and creep mechanism of Earth's inner core Geophys. Res. Lett. 31 (20).

Vočadlo, L., 2007. Mineralogy of the earth-the earth's core: iron and iron alloys. Treatise Geophys. 2, 91-120.

Weber, P., Machetel, P., 1992. Convection within the innercore and thermal implications. Geophys. Res. Lett. 19 (21), 2107-2110.

Wenk, H.R., Baumgardner, J.R., Lebensohn, R., Tomé, C.N., 2000. A convection mode to explain anisotropy of the inner core. J. Geophys. Res. 105 (B3), 5663-5677.

Woodhouse, J.H., Giardini, D., Li, X., 1986. Evidence for inner core anisotropy from free oscillations. Geophys. Res. Lett. 13, 1549-1552.

Yoshida, S., Sumita, I., Kumazawa, M., 1996. Growth model of the inner core coupled with the outer core dynamics and the resulting elastic anisotropy. J. Geophys. Res. 101 (B12), 28085-28103.

Yukutake, T., 1998. Implausibility of thermal convection in the Earth's solid inner core. Phys. Earth Planet. Inter. 108 (1), 1-13. 